\begin{tabular}{|c|l|}
\hline Title & The null condition and global existence of solutions to systems of wave equations with different speeds \\
\hline Author(s) & Agemi, R.; Yokoyama, K. \\
\hline Citation & Hokkaido University Preprint Series in Mathematics, 349, 1-42 \\
\hline Issue Date & 1996-9-1 \\
\hline DOI & 10.14943/83495 \\
\hline Doc URL & http://hdl.handle.net/2115/69099 \\
\hline Type & bulletin (article) \\
\hline File Information & pre349.pdf \\
\hline
\end{tabular}

Instructions for use 
The Null Condition and Global Existence of Solutions to Systems of Wave Equations with Different Speeds

R. Agemi and K. Yokoyama

Series \#349. September 1996 


\section{HOKKAIDO UNIVERSITY PREPRINT SERIES IN MATHEMATICS}

\#324 A. Arai, Factorization of self-adjoint operators by abstract Dirac operators and its application to second quantizations on Boson Fermion Fock spaces, 15 pages. 1995.

\#325 K. Sugano, On strongly separable Frobenius extensions, 11 pages. 1995.

\#326 D. Lehmann and T. Suwa, Residues of holomorphic vector fields on singular varieties, 21 pages. 1995.

\#327 K. Tsutaya, Local regularity of non-resonant nonlinear wave equations, 23 pages. 1996.

\#328 T. Ozawa and Y. Tsutsumi, Space-time estimates for null gauge forms and nonlinear Schrödinger equations, 25 pages. 1996.

\#329 O. Ogurisu, Anticommutativity and spin 1/2 Schrödinger operators with magnetic fields, 12 pages. 1996.

\#330 Y. Kurokawa, Singularities for projections of contour lines of surfaces onto planes, 24 pages. 1996.

\#331 M.-H. Giga and Y. Giga, Evolving graphs by singular weighted curvature, 94 pages. 1996.

\#332 M. Ohnuma and K. Sato; Singular degenerate parabolic equations with applications to the p-laplace diffusion equation, 20 pages. 1996.

\#333 T. Nakazi, The spectra of Toeplitz operators with unimodular symbols, 9 pages. 1996.

\#334 B. Khanedani and T. Suwa, First variation of horomorphic forms and some applications, 11 pages. 1996.

\#335 J. Seade and T. Suwa, Residues and topological invariants of singular holomorphic foliations ${ }^{1}, 28$ pages. 1996.

\#336 Y. Giga, M.E. Gurtin and J. Matias, On the dynamics of crystalline motions, 67 pages. 1996.

\#337 I. Tsuda, A new type of self-organization associated with chaotic dynamics in neural networks, 22 pages. 1996.

\#338 F. Hiroshima, A scaling limit of a Hamiltonian of many nonrelativistic particles interacting with a quantized radiation field, 34 pages. 1996.

\#339 N. Tominaga, Analysis of a family of strongly commuting self-adjoint operators with applications to perturbed Dirac operators, 29 pages. 1996.

\#340 A. Inoue, Abel-Tauber theorems for Fourier-Stieltjes coefficients, 17 pages. 1996.

\#341 G. Ishikawa, Topological classification of the tangent developables of space curves, 19 pages. 1996.

\#342 Y. Shimizu, A remark on estimates of bilinear forms of gradients in Hardy space, 8 pages. 1996.

\#343 N. kawazumi and S. Morita, The primary approximation to the cohomology of the moduli space of curves and cocycles for the stable characteristic classes, 11 pages. 1996.

\#344 M.-H. Giga and Y. Giga, A subdifferential interpretation of crystalline motion under nonuniform driving force, 18 pages. 1996.

\#345 A. Douai and H. Terao, The determinant of a hypergeometric period matrix, 20 pages. 1996.

\#346 H. Kubo and K. Kubota, Asymptotic behaviors of radially symmetric solutions of $\square u=|u|^{p}$ for super critical values $p$ in even space dimensions, 66 pages. 1996.

\#347 T. Nakazi and T. Yamamoto, Weighted Norm Inequalities For Some Singular Integral Operators, 17 pages. 1996.

\#348 Y. Ito and I. Nakamura, Hilbert schemes and simple singularities $A_{n}$ and $D_{n}, 22$ pages. 1996 


\title{
The Null Condition and Global Existence of Solutions to Systems of Wave Equations with Different Speeds
}

\author{
Rentaro Agemi and Kazuyoshi Yokoyama \\ Department of Mathematics, Hokkaido University, \\ Sapporo 060, Japan
}

\begin{abstract}
In this paper, we consider the initial value problems to systems of quasilinear wave equations with different speeds in two space dimensions. Applying John-Shatah observations to our problem, we introduce the null condition for the system with different speeds. Moreover, we prove a global existence theorem for a class satisfying the null condition.
\end{abstract}

\section{Introduction.}

We shall start this paper with the description of John-Shatah observations on the null condition. We consider the scalar quasilinear wave equations with quadratic nonlinearity in three space dimensions. Introducing the space-time gradient of unknown, one can find that components of the gradient satisfy some hyperbolic system of first order. The plane wave solutions of this system satisfy hyperbolic systems of first order in one space dimension. Making use of the results in F. John[3], F. John and J. Shatah have proved in F. John[5] the following 
remarkable fact: The requirement that no plane wave solution of this system is genuinely nonlinear leads to a class of equations which satisfy the null condition (S. Klainerman[9]).

We apply John-Shatah observations to a system of quasilinear wave equations with different speeds in two space dimensions. We consider the system with unknown vector $u(t, x)={ }^{t}\left(u^{1}(t, x), \ldots, u^{m}(t, x)\right)$ in the form

$$
\partial_{t}^{2} u^{i}-c_{i}^{2} \Delta u^{i}=\sum_{j=1}^{m} \sum_{\alpha, \beta=0}^{2} C_{i j}^{\alpha \beta}(\partial u) \partial_{\alpha} \partial_{\beta} u^{j} \quad(i=1, \ldots, m)
$$

where $\partial u$ stands for space-time gradient of $u$, i.e.

$$
\begin{aligned}
& \partial u={ }^{t}\left(\partial u^{1}, \ldots, \partial u^{m}\right) \\
& \partial u^{i}={ }^{t}\left(\partial_{0} u^{i}, \partial_{1} u^{i}, \partial_{2} u^{i}\right) \\
& \partial_{0}=\frac{\partial}{\partial t}, \partial_{1}=\frac{\partial}{\partial x_{1}}, \partial_{2}=\frac{\partial}{\partial x_{2}}
\end{aligned}
$$

and the $c_{i}(i=1, \ldots, m)$ are positive constants different from each other. We assume that $C_{i j}^{\alpha \beta}$ are $C^{\infty}$-functions of their arguments which vanish at $\partial u=0$ of second order. Set $v=\partial u$. Then one can find from (1.1) and (1.2) that the vector $v$ satisfies the system of first order which is hyperbolic near $v=0$ :

$$
\sum_{\alpha=0}^{2} a^{\alpha}(v) \partial_{\alpha} v=0
$$

For the concrete expression of $a^{\alpha}(v)$ see section 2 . We next consider the plane wave solutions $w$ of the system (1.3):

$$
v(t, x)=w(t, s), s=\sum_{i=1}^{2} \zeta_{i} x_{i}
$$

where $\zeta=\left(\zeta_{1}, \zeta_{2}\right) \in \mathbf{R}^{2}$ and $\zeta \neq 0$. Then one can find from (1.3) and (1.4) that the vector $w$ satisfies the system in one space dimension:

$$
a^{0}(w) \partial_{t} w+\sum_{i=1}^{2} \zeta_{i} a^{i}(w) \partial_{s} w=0
$$

We take the initial values for the solutions $w$ of (1.5) in the form

$$
w(0, s)=\varepsilon \varphi(s)
$$


where $\varphi$ has compact support and $\varepsilon$ is small positive constant.

Since the system (1.1) has the cubic nonlinearity, the system (1.5) is not genuinely nonlinear. Thus it is natural to require that the lifespan $T_{\varepsilon}$ of solutions to (1.5) and (1.6) is at least of order $\varepsilon^{-3}$ for any $\zeta$. Making use of the results in Li Ta-tsien, Kong De-xing and Zhou Yi[12], we shall prove in section 2 that the requirement above is equivalent to the following fact: it holds that

$$
\left.\sum_{\alpha, \beta, \gamma, \delta=0}^{2} \frac{\partial^{2} C_{i i}^{\alpha \beta}}{\partial\left(\partial_{\gamma} u^{i}\right) \partial\left(\partial_{\delta} u^{i}\right)}\right|_{\partial u=0} X_{\alpha}^{i} X_{\beta}^{i} X_{\gamma}^{i} X_{\delta}^{i}=0 \quad(i=1, \ldots, m)
$$

for any real vector $X^{i}=\left(X_{0}^{i}, X_{1}^{i}, X_{2}^{i}\right)$ satisfying

$$
\left(X_{0}^{i}\right)^{2}-c_{i}^{2} \sum_{j=1}^{2}\left(X_{j}^{i}\right)^{2}=0 .
$$

Thus we can interpret this as the null condition for the system (1.1) with different speeds. If

$$
\left.\frac{\partial^{2} C_{i i}^{\alpha \beta}}{\partial\left(\partial_{\gamma} u^{i}\right) \partial\left(\partial_{\delta} u^{i}\right)}\right|_{\partial u=0}=0 \quad\left(\begin{array}{c}
i=1, \ldots, m \\
\alpha, \beta, \gamma, \delta=0,1,2
\end{array}\right)
$$

then the null condition (1.7) is automatically satisfied. The main aim of this paper is to prove the global existence of solution to (1.1) with small data under the assumption (1.9). It is still open whether the null condition (1.7) guarantees the global existence of solution to (1.1).

The null condition for the nonlinear elastic wave equation is more complicated comparing with (1.7). T. Sideris has studied in [13] a class satisfying the null condition.

In section 3 , we introduce some notations and in section 4 we state the main result. In section 5, using the representation of solution to (1.1) in Kovalyov[10], we estimate the first order derivatives of solution. Finally we prove the main results in section 7 using the estimates and energy inequalities in section 6 .

\section{The null condition.}

In this section we introduce the null condition for the system (1.1) with different speeds stated in Introduction. 
We consider the system in the form

$$
\sum_{j=1}^{m} \sum_{\alpha, \beta=0}^{2} a_{i j}^{\alpha \beta}(\partial u) \partial_{\alpha} \partial_{\beta} u^{j}=0 \quad(i=1, \ldots, m)
$$

where $\partial u$ stands for space-time gradient of $u$

$$
\begin{aligned}
& \partial u={ }^{t}\left(\partial u^{1}, \ldots, \partial u^{m}\right), \\
& \partial u^{i}={ }^{t}\left(\partial_{0} u^{i}, \partial_{1} u^{i}, \partial_{2} u^{i}\right), \\
& \partial_{0}=\frac{\partial}{\partial t}, \partial_{1}=\frac{\partial}{\partial x_{1}}, \partial_{2}=\frac{\partial}{\partial x_{2}} .
\end{aligned}
$$

We assume that

$$
\begin{aligned}
& a_{i j}^{\alpha \beta}=a_{i j}^{\beta \alpha}, a_{i i}^{00}(0)=1, \\
& a_{i j}^{\alpha \beta}(0)=-c_{i}^{2} \delta_{i j} \delta_{\alpha \beta} \text { for }(\alpha, \beta) \neq(0,0), \\
& c_{i}>0, c_{i} \neq c_{j} \text { for } i \neq j
\end{aligned}
$$

and $a_{i i}^{00}(\partial u)-1, a_{i j}^{\alpha \beta}(\partial u)+c_{i}^{2} \delta_{i j} \delta_{\alpha \beta}$ vanish at $\partial u=0$ at least of second order, that is,

$$
\begin{aligned}
& a_{i i}^{00}(\partial u)=1+O\left(|\partial u|^{2}\right) \\
& a_{i j}^{\alpha \beta}(\partial u)=-c_{i}^{2} \delta_{i j} \delta_{\alpha \beta}+O\left(|\partial u|^{2}\right)
\end{aligned}
$$

near $\partial u=0$.

Set

$$
\begin{aligned}
v & ={ }^{t}\left(v^{1}, \ldots, v^{m}\right) \\
v^{i} & ={ }^{t}\left(v_{0}^{i}, v_{1}^{i}, v_{2}^{i}\right), v_{\alpha}^{i}=\partial_{\alpha} u^{i} .
\end{aligned}
$$

Then we find from (2.1) and (2.4) that the vector $v$ satisfies a system of first order which is hyperbolic near $v=0$ :

$$
\sum_{\alpha=0}^{2} a^{\alpha}(v) \partial_{\alpha} v=0
$$

Here the $3 m \times 3 m$ matrices $a^{\alpha}$ are defined by

$$
a^{\alpha}=\left(A_{i j}^{\alpha}: i, j=1, \ldots, m\right),
$$


where

$$
\begin{aligned}
& A_{i j}^{0}=\left(\begin{array}{ccc}
a_{i j}^{00} & & 0 \\
& \delta_{i j} & \\
0 & & \delta_{i j}
\end{array}\right), A_{i j}^{1}=\left(\begin{array}{ccc}
2 a_{i j}^{10} & a_{i j}^{11} & a_{i j}^{12} \\
-\delta_{i j} & 0 \\
0 & 0
\end{array}\right) \text {, } \\
& A_{i j}^{2}=\left(\begin{array}{ccc}
2 a_{i j}^{20} & a_{i j}^{21} & a_{i j}^{22} \\
0 & 0 \\
-\delta_{i j} &
\end{array}\right)
\end{aligned}
$$

We next consider the plane wave solution $v(t, x)$ of the equation $(2.5)$ :

$$
v(t, x)=w(t, s), s=\sum_{i=1}^{2} \zeta_{i} x_{i}
$$

where $\zeta=\left(\zeta_{1}, \zeta_{2}\right) \in \mathbf{R}^{2}$ and $\zeta \neq 0$. Then we find from (2.5) and (2.8) that the vector $w$ satisfies a system in one space dimension.

$$
\partial_{t} w+a(w) \partial_{s} w=0
$$

where

$$
a(w)=a^{0}(w)^{-1} \sum_{i=1}^{2} \zeta_{i} a^{i}(w) .
$$

We take the initial values for the solution $w$ of (2.9) in the form

$$
w(0, s)=\varepsilon \varphi(s)
$$

where $\varphi$ has compact support and $\varepsilon>0$.

We shall seek the eigenvalues $\lambda=\lambda(w)$ of the matrix $a(w)$ and the corresponding eigenvector $\xi=\xi(w)$. By the definition, $\lambda$ satisfies an equation

$$
\operatorname{det}\left(\lambda a^{0}(w)-\sum_{i=1}^{2} \zeta_{i} a^{i}(w)\right)=0
$$

We can verify by induction that

$$
\operatorname{det}\left(\lambda a^{0}(w)-\sum_{i=1}^{2} \zeta_{i} a^{i}(w)\right)=\lambda^{m} \operatorname{det}\left(p_{i j}: i, j=1, \ldots, m\right)
$$


where

$$
p_{i j}=a_{i j}^{00} \lambda^{2}-2 \lambda \sum_{k=1}^{2} a_{i j}^{k 0} \zeta_{k}+\sum_{k, l=1}^{2} a_{i j}^{k l} \zeta_{k} \zeta_{l} .
$$

Therefore we find from (2.2), (2.3), (2.12), (2.13) and (2.14) that the eigenvalues $\lambda_{i}^{ \pm}(0)(i=$ $1, \ldots, m)$ of the matrix $a(0)$, aside from the trivial multiple eigenvalue $\lambda=0$, become

$$
\lambda_{i}^{ \pm}(0)= \pm c_{i}|\zeta|,|\zeta|=\left(\zeta_{1}^{2}+\zeta_{2}^{2}\right)^{1 / 2}
$$

According to (2.4) we arrange the components of a vector $\xi \in \mathbf{R}^{3 m}$ as follows:

$$
\begin{aligned}
\xi & ={ }^{t}\left(\xi^{1}, \ldots, \xi^{m}\right) \\
\xi^{i} & ={ }^{t}\left(\xi_{0}^{i}, \xi_{1}^{i}, \xi_{2}^{i}\right)
\end{aligned}
$$

Then we find from $(2.2),(2.3),(2.7)$ and $(2.12)$ that the eigenvector $\xi_{i}^{\ddagger}(0)$ corresponding to $\lambda_{i}^{ \pm}(0)$ becomes

$$
\begin{aligned}
& \left(\xi_{i}^{ \pm}(0)\right)^{j}={ }^{t}(0,0,0) \text { for } j \neq i \\
& \left(\xi_{i}^{ \pm}(0)\right)^{i}={ }^{t}\left(\mp 1, \zeta_{1} / c_{i}|\zeta|, \zeta_{2} / c_{i}|\zeta|\right)
\end{aligned}
$$

and the eigenvectors $\xi_{i}(i=1, \ldots, m)$ corresponding to the trivial eigenvalue 0 become

$$
\begin{aligned}
& \left(\xi_{i}\right)^{j}={ }^{t}(0,0,0) \text { for } j \neq i \\
& \left(\xi_{i}\right)^{j}={ }^{t}\left(0, \zeta_{2},-\zeta_{1}\right) .
\end{aligned}
$$

Since $\xi_{i}^{ \pm}(0), \xi_{i}(i=1, \ldots, m)$ are linearly independent, we see that the system (2.9) is hyperbolic near $w=0$.

We now require that a solution $w(t, s)$ to the initial value problem $(2.9),(2.11)$ has a lifespan $T_{\varepsilon}$ which is at least of order $\varepsilon^{-3}$ for any $\zeta \in \mathbf{R}^{2}$. This requirement is equivalent to the following facts[12]:

$$
\left.\sum_{j=1}^{m} \sum_{\alpha=0}^{2} \frac{\partial \lambda_{i}^{ \pm}}{\partial w_{\alpha}^{j}}\right|_{w=0}\left(\xi_{i}^{ \pm}(0)\right)_{\alpha}^{j}=0
$$

and

(2.19) $\left.\sum_{j, k=1}^{m} \sum_{\alpha, \beta=0}^{2} \frac{\partial^{2} \lambda_{i}^{ \pm}}{\partial w_{\alpha}^{j} \partial w_{\beta}^{k}}\right|_{w=0}\left(\xi_{i}^{ \pm}(0)\right)_{\alpha}^{j}\left(\xi_{i}^{ \pm}(0)\right)_{\beta}^{k}=0 \quad$ for $\quad i=1, \ldots, m$ and $\zeta \in \mathbf{R}^{2}$. 
Set

$$
P(\lambda)=\operatorname{det}\left(p_{i j}(\lambda): i, j=1, \ldots, m\right)
$$

Differentiating the equations

$$
P\left(\lambda_{i}^{ \pm}(w)\right)=0 \quad(i=1, \ldots, m)
$$

in a variable $w_{\alpha}^{j}$ and evaluating the results at $w=0$, we get

$$
\left.2 c_{i}|\zeta|^{2 m-1} \prod_{l \neq i}\left(c_{i}^{2}-c_{l}^{2}\right) \frac{\partial \lambda_{i}^{ \pm}}{\partial w_{\alpha}^{j}}\right|_{w=0}=0
$$

which implies

$$
\left.\frac{\partial \lambda_{i}^{ \pm}}{\partial w_{\alpha}^{j}}\right|_{w=0}=0
$$

for all $i, j, \alpha$. Therefore it follows from (2.2), (2.3), (2.14) and (2.21) that the condition (2.18) holds trivially and

$$
\left.\frac{\partial\left(p_{i j}\left(\lambda_{k}^{ \pm}\right)\right)}{\partial w_{\alpha}^{l}}\right|_{w=0}=0
$$

for all $i, j, k, l, \alpha$. Next differentiating twice the equations (2.20) in variables $w_{\alpha}^{j}$ and $w_{\beta}^{k}$ and evaluating the results at $w=0$, we get

$$
\left.|\zeta|^{2 m-2} \prod_{l \neq i}\left(c_{i}^{2}-c_{l}^{2}\right) \frac{\partial^{2}\left(p_{i i}\left(\lambda_{i}^{ \pm}\right)\right)}{\partial w_{\alpha}^{j} \partial w_{\beta}^{k}}\right|_{w=0}=0
$$

for all $i, j, k, \alpha, \beta$. By the definition (2.14) of $p_{i i}(\lambda)$, we have

$$
\begin{aligned}
\left.\frac{\partial^{2}\left(p_{i i}\left(\lambda_{i}^{ \pm}\right)\right)}{\partial w_{\alpha}^{j} \partial w_{\beta}^{k}}\right|_{w=0}= & \left.2 \lambda_{i}^{ \pm}(0) \frac{\partial^{2} \lambda_{i}^{ \pm}}{\partial w_{\alpha}^{j} \partial w_{\beta}^{k}}\right|_{w=0}+\left.\lambda_{i}^{ \pm}(0)^{2} \frac{\partial^{2} a_{i i}^{00}}{\partial w_{\alpha}^{j} \partial w_{\beta}^{k}}\right|_{w=0} \\
& -\left.2 \lambda_{i}^{ \pm}(0) \sum_{l=1}^{2} \frac{\partial^{2} a_{i i}^{l 0}}{\partial w_{\alpha}^{j} \partial_{\beta}^{k}}\right|_{w=0} \zeta_{l}+\left.\sum_{h, l=1}^{2} \frac{\partial a_{i i}^{h l}}{\partial w_{\alpha}^{j} \partial w_{\beta}^{k}}\right|_{w=0} \zeta_{h} \zeta_{l} .
\end{aligned}
$$

Then it follows from $(2.15),(2.17),(2.23)$ and (2.24) that

$$
\left.\frac{\partial^{2} \lambda_{i}^{ \pm}}{\partial w_{\alpha}^{j} \partial w_{\beta}^{k}}\right|_{w=0}=\left.\frac{\mp c_{i}|\zeta|}{2} \sum_{\gamma, \delta=0}^{2} \frac{\partial^{2} a_{i i}^{\gamma \delta}}{\partial w_{\alpha}^{j} \partial w_{\beta}^{k}}\right|_{w=0}\left(\xi_{i}^{ \pm}(0)\right)_{\gamma}^{i}\left(\xi_{i}^{ \pm}(0)\right)_{\delta}^{i}
$$


Therefore we find from (2.17) and (2.25) that the condition (2.19) is equivalent to

$$
\left.\sum_{\alpha, \beta, \gamma, \delta=0}^{2} \frac{\partial^{2} a_{i i}^{\gamma \delta}}{\partial w_{\alpha}^{i} \partial w_{\beta}^{i}}\right|_{w=0}\left(\xi_{i}^{ \pm}(0)\right)_{\alpha}^{i}\left(\xi_{i}^{ \pm}(0)\right)_{\beta}^{i}\left(\xi_{i}^{ \pm}(0)\right)_{\gamma}^{i}\left(\xi_{i}^{ \pm}(0)\right)_{\delta}^{i}=0
$$

for $i=1, \ldots, m$ and $\zeta \in \mathbf{R}^{2}$. By the definition (2.17) of $\left(\xi_{i}^{ \pm}(0)\right)^{i}$, we have

$$
\left\{\left(\xi_{i}^{ \pm}(0)\right)_{0}^{i}\right\}^{2}-c_{i}^{2} \sum_{j=1}^{2}\left\{\left(\xi_{i}^{ \pm}(0)\right)_{j}^{i}\right\}^{2}=0
$$

for all $\zeta \in \mathbf{R}^{2}$.

Consequently we have proved the following

Proposition 2.1 The lifespan $T_{\varepsilon}$ of a unique solution $w(t, s)$ of the initial value problem (2.9), (2.11) is at least of order $\varepsilon^{-3}$ for any $\zeta \in \mathbf{R}^{2}$ if and only if it holds that

$$
\left.\sum_{\alpha, \beta, \gamma, \delta=0}^{2} \frac{\partial^{2} a_{i i}^{\gamma \delta}}{\partial w_{\alpha}^{i} \partial w_{\beta}^{i}}\right|_{w=0} X_{\alpha}^{i} X_{\beta}^{i} X_{\gamma}^{i} X_{\delta}^{i}=0 \quad(i=1, \ldots, m)
$$

for all real vector $X^{i}=\left(X_{0}^{i}, X_{1}^{i}, X_{2}^{i}\right)$ satisfying

$$
\left(X_{0}^{i}\right)^{2}-c_{i}^{2} \sum_{j=1}^{2}\left(X_{j}^{i}\right)^{2}=0
$$

Setting

$$
\begin{aligned}
& C_{i i}^{00}(\partial u)=1-a_{i i}^{00}(\partial u) \\
& C_{i j}^{\alpha \beta}(\partial u)=-c_{i}^{2} \delta_{i j} \delta_{\alpha \beta}-a_{i j}^{\alpha \beta}(\partial u) \quad(\alpha, \beta) \neq(0,0)
\end{aligned}
$$

we see that the null condition (1.7), (1.8) follows from Proposition 2.1.

\section{Notations.}

To begin with, we introduce some notations that are used throughout the paper.

Partial derivatives are denoted by

$$
\partial_{0}=\partial_{t}=\frac{\partial}{\partial t}, \partial_{1}=\frac{\partial}{\partial x_{1}}, \partial_{2}=\frac{\partial}{\partial x_{2}}
$$


We also use the angular derivative:

$$
\Omega=x_{1} \partial_{2}-x_{2} \partial_{1}
$$

We set

$$
\mathcal{D}=\left(\mathcal{D}_{1}, \mathcal{D}_{2}, \mathcal{D}_{3}\right)=\left(\partial_{1}, \partial_{2}, \Omega\right)
$$

and define

$$
\mathcal{D}^{A}=\mathcal{D}_{1}^{A_{1}} \mathcal{D}_{2}^{A_{2}} \mathcal{D}_{3}^{A_{3}},|A|=A_{1}+A_{2}+A_{3},
$$

where $A=\left(A_{1}, A_{3}, A_{3}\right)$ is a multi-index.

Let $u={ }^{t}\left(u^{1}, \ldots, u^{m}\right)$ be an unknown vector and set

$$
w_{i}(t, r)=(r+1)^{1 / 2-\gamma}(t+r+1)^{\gamma}\left(\left|r-c_{i} t\right|+1\right)^{1 / 2}
$$

for $0<\gamma<1 / 2$. Then we define, for a non-negative integer $k$,

$$
\begin{aligned}
|\partial u(t, x)| & =\sum_{i=1}^{m} \sum_{\alpha=0}^{2}\left|\partial_{\alpha} u^{i}(t, x)\right| \\
|\partial u(t)|_{k} & =\sum_{|A| \leq k} \sum_{i=1}^{m} \sum_{\alpha=0}^{2} \sup _{x \in \mathbf{R}^{2}}\left|\mathcal{D}^{A} \partial_{\alpha} u^{i}(t, x)\right| \\
\|\partial u(t)\|_{k} & =\sum_{|A| \leq k} \sum_{i=1}^{m} \sum_{\alpha=0}^{2}\left\|\mathcal{D}^{A} \partial_{\alpha} u^{i}(t, \cdot)\right\|_{L^{2}\left(\mathbf{R}^{2}\right)} \\
{[\partial u(t)]_{k} } & =\sum_{|A| \leq k} \sum_{i=1}^{m} \sum_{\alpha=0}^{2} \sup _{x \in \mathbf{R}^{2}}\left|w_{i}(t,|x|) \mathcal{D}^{A} \partial_{\alpha} u^{i}(t, x)\right| .
\end{aligned}
$$

Moreover, we define

$$
\begin{aligned}
|\partial u|_{k}(t) & =\sup _{0<s<t}|\partial u(s)|_{k}, \\
{[\partial u]_{k}(t) } & =\sup _{0<s<t}[\partial u(s)]_{k} .
\end{aligned}
$$

In what follows, $M$ denotes various constant depending on $F_{i}, f^{i}, g^{i}$ and $c_{i}$.

\section{Statement of the Main Result.}

The initial value problem to be considered is

$$
\left\{\begin{array}{lll}
\partial_{t}^{2} u^{i}-c_{i}^{2} \Delta u^{i}=F_{i}\left(\partial u, \partial^{2} u\right) & \text { in }[0, \infty) \times \mathbf{R}^{2} \\
u^{i}(0, \cdot)=\varepsilon f^{i}, \partial_{t} u^{i}(0, \cdot)=\varepsilon g^{i} & \text { in } \mathbf{R}^{2}
\end{array} \quad(i=1, \ldots, m)\right.
$$


where $c_{i}$ are positive constants and $\varepsilon>0$ is small parameter. Moreover, $f^{i}$ and $g^{i}$ are $C^{\infty}$ functions with compact support. We describe some assumptions on the initial value problem (4.1) and state the main theorem.

First, we assume that $F_{i}$ are of first degree with respect to the second derivatives of $u$ :

$$
F_{i}\left(\partial u, \partial^{2} u\right)=\sum_{j=1}^{m} \sum_{\alpha, \beta=0}^{2} C_{i j}^{\alpha \beta}(\partial u) \partial_{\alpha} \partial_{\beta} u^{j}+E_{i}(\partial u) .
$$

Here, $C_{i j}^{\alpha \beta}$ and $E_{i}$ are $C^{\infty}$-functions of $\partial u$ in $\{|\partial u|<1\}$ that satisfy

$$
\begin{aligned}
& C_{i j}^{\alpha \beta}=C_{i j}^{\beta \alpha}=C_{j i}^{\alpha \beta}, \\
& \left|C_{i j}^{\alpha \beta}(\partial u)\right| \leq M|\partial u|^{2}, \\
& \left|E_{i}(\partial u)\right| \leq M|\partial u|^{3} .
\end{aligned}
$$

Assuming (4.2)-(4.5), M. Kovalyov[10] proved the almost global existence of the solution to (4.1).

Second, we assume the null condition (1.9) for different speeds introduced in Introduction:

$$
\begin{aligned}
& c_{i} \neq c_{j} \quad \text { for } \quad i \neq j \\
& \left.\frac{\partial^{2} C_{i i}^{\alpha \beta}}{\partial\left(\partial_{\gamma} u^{i}\right) \partial\left(\partial_{\delta} u^{i}\right)}\right|_{\partial u=0}=0 \quad\left(\begin{array}{c}
i=1, \ldots, m \\
\alpha, \beta, \gamma, \delta=0,1,2
\end{array}\right) .
\end{aligned}
$$

The condition for $E_{i}$ are

$$
\left.\frac{\partial^{3} E_{i}}{\partial\left(\partial_{\alpha} u^{i}\right) \partial\left(\partial_{\beta} u^{i}\right) \partial\left(\partial_{\gamma} u^{i}\right)}\right|_{\partial u=0}=0 \quad\left(\begin{array}{c}
i=1, \ldots, m \\
\alpha, \beta, \gamma=0,1,2
\end{array}\right)
$$

in accordance with (4.7).

Theorem Let us assume (4.2)-(4.8). Then there exists a positive constant $\varepsilon_{0}$ depending on given functions such that the initial value problem (4.1) has a unique $C^{\infty}$ - solution in $[0, \infty) \times \mathbf{R}^{2}$ for all $\varepsilon$ with $0<\varepsilon<\varepsilon_{0}$.

M. Kovalyov showed in [11] that the theorem holds when $C_{i j}^{\alpha \beta}=0$ and $E_{i}(i=1, \ldots, m)$ satisfy the condition

$$
\left.\frac{\partial^{3} E_{i}}{\partial\left(\partial_{\alpha} u^{j}\right) \partial\left(\partial_{\beta} u^{j}\right) \partial\left(\partial_{\gamma} u^{j}\right)}\right|_{\partial u=0}=0 \quad\left(\begin{array}{c}
i, j=1, \ldots, m \\
\alpha, \beta, \gamma=0,1,2
\end{array}\right)
$$


instead of (4.8).

\section{Estimate of the First Derivatives of the Solutions to Initial Value Problems.}

The aim of this section is to estimate the first derivatives of the solution to the initial value problem:

$$
\left\{\begin{array}{lll}
\partial_{t}^{2} u-\Delta u=F(t, x) & \text { in } & {[0, T) \times \mathbf{R}^{2}} \\
u(0, \cdot)=\partial_{t} u(0, \cdot)=0 & \text { in } \quad \mathbf{R}^{2}
\end{array}\right.
$$

Here, $F$ is a $C^{\infty}$ function in $[0, T) \times \mathbf{R}^{2}$. For this purpose, we use the representation formula of the solution to (5.1) which has proved by M. Kovalyov[10]:

Proposition 5.1 Let $u \in C^{\infty}\left([0, T) \times \mathbf{R}^{2}\right)$ be the solution of the initial value problem (5.1). Then, $u$ has the following representation:

$$
\begin{aligned}
u(t, x)= & \frac{1}{2 \pi} \iint_{D^{\prime}} r d r d s \int_{-\varphi}^{\varphi} K_{1} F\left(s, r e^{\sqrt{-1}(\theta+\psi)}\right) d \psi \\
& +\frac{1}{2 \pi} \chi(t-a) \iint_{D^{\prime \prime}} r d r d s \int_{-\pi}^{\pi} K_{1} F\left(s, r e^{\sqrt{-1}(\theta+\psi)}\right) d \psi
\end{aligned}
$$

where

$$
\begin{aligned}
x & =(a \cos \theta, a \sin \theta)=a e^{\sqrt{-1} \theta} \\
\varphi & =\arccos \frac{a^{2}+r^{2}-(t-s)^{2}}{2 a r} \text { for }(s, r) \in D^{\prime} \\
K_{1} & =\frac{1}{\left\{(t-s)^{2}-a^{2}-r^{2}+2 a r \cos \psi\right\}^{1 / 2}} \\
\chi(s) & = \begin{cases}1 & (s>0) \\
0 & (s \leq 0)\end{cases}
\end{aligned}
$$

Moreover, the domains $D^{\prime}$ and $D^{\prime \prime}$ are defined as follows.

$$
\begin{aligned}
D^{\prime} & =\left\{(s, r) \mid 0<s<t, r_{1}<r<r_{2}\right\} \\
D^{\prime \prime} & = \begin{cases}\left\{(s, r) \mid 0<s<t-a, 0<r<r_{1}\right\} & \text { for } t>a \\
\emptyset & \text { for } t \leq a\end{cases}
\end{aligned}
$$


where

$$
r_{1}=|a-t+s|, r_{2}=a+t-s
$$

Next, we derive representation formulae for the first derivatives of the solution of the initial value problem (5.1) from Proposition 5.1. In order to present the formulae, we set

$$
\begin{aligned}
& \delta=\min \{1 / 2, a\} \\
& \tilde{\delta}=\min \{1 / 2,(t-a) / 2\} \\
& (a=|x|)
\end{aligned}
$$

and split the domains $D^{\prime}$ and $D^{\prime \prime}$ as follows:

$$
\begin{aligned}
& D^{\prime}= \text { blue } \cup \text { white } \\
& \text { blue }=\left\{(s, r) \in D^{\prime} \mid r_{1}<r \leq r_{1}+\delta \text { or } r_{2}-\delta \leq r<r_{2}\right\} \text { white }= \begin{cases}D^{\prime} \backslash \text { blue } & \text { for } \delta=1 / 2 \\
\emptyset & \text { for } \delta=a\end{cases} \\
& D^{\prime \prime}=\text { black } \cup \text { red } \text { black }=\left\{(s, r) \in D^{\prime \prime} \mid r_{1}-\tilde{\delta} \leq r<r_{1} \text { or } 0<r \leq \tilde{\delta}\right\} \\
& \text { red }= \begin{cases}D^{\prime \prime} \backslash \text { black } & \text { for } \tilde{\delta}=1 / 2 \\
\emptyset & \text { for } \tilde{\delta}=(t-a) / 2\end{cases}
\end{aligned}
$$

We set

$$
\begin{aligned}
I_{\text {blue }}(F)(t, x) & =\iint_{\text {blue }} r d r d s \int_{-\varphi}^{\varphi} K_{1} F\left(s, r e^{\sqrt{-1}(\theta+\psi)}\right) d \psi \\
I_{\text {white }}(F)(t, x) & =\iint_{\text {white }} r d r d s \int_{-\varphi}^{\varphi} K_{1} F\left(s, r e^{\sqrt{-1}(\theta+\psi)}\right) d \psi \\
I_{\text {black }}(F)(t, x) & =\iint_{\text {black }} r d r d s \int_{-\pi}^{\pi} K_{1} F\left(s, r e^{\sqrt{-1}(\theta+\psi)}\right) d \psi \\
I_{\text {red }}(F)(t, x) & =\iint_{\text {red }} r d r d s \int_{-\pi}^{\pi} K_{1} F\left(s, r e^{\sqrt{-1}(\theta+\psi)}\right) d \psi
\end{aligned}
$$

Then, by Proposition 5.1, $\partial_{\mu} u(\mu=0,1,2)$ is represented as

$$
\begin{aligned}
\partial_{\mu} u= & \frac{1}{2 \pi}\left\{I_{b l u e}\left(\partial_{\mu} F\right)+\chi(a-1 / 2) I_{\text {white }}\left(\partial_{\mu} F\right)\right. \\
& \left.+\chi(t-a) I_{\text {black }}\left(\partial_{\mu} F\right)+\chi(t-a-1) I_{\text {red }}\left(\partial_{\mu} F\right)\right\}
\end{aligned}
$$


Following [10], we change the variable of integration from $\psi$ to $\tau$ by the map $\psi=\Psi$, where

$$
\begin{aligned}
& \Psi=\arccos [1+P \tau-\tau] \\
& P=\frac{a^{2}+r^{2}-(t-s)^{2}}{2 a r}
\end{aligned}
$$

Then we have the following

\section{Proposition 5.2}

$$
\begin{aligned}
I_{\text {white }}\left(\partial_{\mu} F\right)(t, x)= & \sum_{j, \alpha=0}^{1}\left\{\iint_{\partial(\text { white })} d \sigma \int_{0}^{1} r K_{2} a_{\mu}^{\alpha}\left(\theta+\Psi_{j}\right) n_{\alpha} F\left(s, r e^{\sqrt{-1}\left(\theta+\Psi_{j}\right)}\right) d \tau\right. \\
& -\iint_{w h i t e} d r d s \int_{0}^{1} \nabla_{\alpha}\left\{r K_{2} a_{\mu}^{\alpha}\left(\theta+\Psi_{j}\right)\right\} F\left(s, r e^{\sqrt{-1}\left(\theta+\Psi_{j}\right)}\right) d \tau \\
& \left.-\iint_{w h i t e} r d r d s \int_{0}^{1} K_{2} a_{\mu}^{\alpha}\left(\theta+\Psi_{j}\right)(\Omega F)\left(s, r e^{\sqrt{-1}\left(\theta+\Psi_{j}\right)}\right) \nabla_{\alpha} \Psi_{j} d \tau\right\} \\
& +\iint_{w h i t e} d r d s \int_{-\varphi}^{\varphi} K_{1} a_{\mu}^{2}(\theta+\psi)(\Omega F)\left(s, r e^{\sqrt{-1}(\theta+\psi)}\right) d \psi \\
I_{r e d}\left(\partial_{\mu} F\right)(t, x)= & \sum_{\alpha=0}^{1} \iint_{\partial(r e d)} r d \sigma \int_{-\pi}^{\pi} K_{1} a_{\mu}^{\alpha}(\theta+\psi) n_{\alpha} F\left(s, r e^{\sqrt{-1}(\theta+\psi)}\right) d \psi \\
& -\sum_{\alpha=0}^{1} \iint_{r e d} d r d s \int_{-\pi}^{\pi} \nabla_{\alpha}\left\{r K_{1}\right\} a_{\mu}^{\alpha}(\theta+\psi) F\left(s, r e^{\sqrt{-1}(\theta+\psi)}\right) d \psi \\
& +\iint_{r e d} d r d s \int_{-\pi}^{\pi} K_{1} a_{\mu}^{2}(\theta+\psi)(\Omega F)\left(s, r e^{\sqrt{-1}(\theta+\psi)}\right) d \psi
\end{aligned}
$$

where

$$
\begin{gathered}
K_{2}=\frac{1}{\{2 a r \tau(1-\tau)(2+P \tau-\tau)\}^{1 / 2}} \\
\Psi_{j}=(-1)^{j} \Psi \\
a_{\mu}^{0}(\theta)=\delta_{\mu}^{0}, \quad a_{\mu}^{1}(\theta)= \begin{cases}0 & (\mu=0) \\
\cos \theta & (\mu=1), \quad a_{\mu}^{2}(\theta)= \begin{cases}0 & (\mu=0) \\
-\sin \theta & (\mu=1) \\
\cos \theta & (\mu=2)\end{cases} \\
\nabla_{\alpha}= \begin{cases}\partial_{s} & (\alpha=0) \\
\partial_{r} & (\alpha=1)\end{cases} \end{cases}
\end{gathered}
$$


$\vec{n}={ }^{t}\left(n_{0}, n_{1}\right)$ is the unit outer normal vector field on $\partial($ white $) \cup \partial(r e d)$, and $d \sigma$ is the line element on $\partial($ white $) \cup \partial($ red $)$.

M. Kovalyov used these formulae in his work[10], but he has omitted the terms containing the first derivatives of $\Psi_{j}$ in the above formulae. So we show the proof for completeness.

Proof. We denote $F\left(s, r e^{\sqrt{-1} \theta}\right)=G(s, r, \theta)$. Then,

$$
\left(\partial_{\mu} F\right)\left(s, r e^{\sqrt{-1} \theta}\right)=\sum_{\alpha=0}^{1} a_{\mu}^{\alpha}(\theta)\left(\nabla_{\alpha} G\right)(s, r, \theta)+\frac{a_{\mu}^{2}(\theta)}{r}\left(\partial_{\theta} G\right)(s, r, \theta) .
$$

Therefore,

$$
\begin{aligned}
I_{w h i t e}\left(\partial_{\mu} F\right)= & \sum_{\alpha=0}^{1} \iint_{w h i t e} r d r d s \int_{-\varphi}^{\varphi} K_{1} a_{\mu}^{\alpha}(\theta+\psi)\left(\nabla_{\alpha} G\right)(s, r, \theta+\psi) d \psi \\
& +\iint_{w h i t e} d r d s \int_{-\varphi}^{\varphi} K_{1} a_{\mu}^{2}(\theta+\psi)\left(\partial_{\theta} G\right)(s, r, \theta+\psi) d \psi
\end{aligned}
$$

Changing variable from $\psi$ to $\tau$ by the map $\psi=\Psi$, we have

$$
\begin{aligned}
& \iint_{w h i t e} r d r d s \int_{-\varphi}^{\varphi} K_{1} a_{\mu}^{\alpha}(\theta+\psi)\left(\nabla_{\alpha} G\right)(s, r, \theta+\psi) d \psi \\
& \quad=\sum_{j=0}^{1} \iint_{w h i t e} r d r d s \int_{0}^{1} K_{2} a_{\mu}^{\alpha}\left(\theta+\Psi_{j}\right)\left(\nabla_{\alpha} G\right)\left(s, r, \theta+\Psi_{j}\right) d \tau
\end{aligned}
$$

Notice that

$$
\left(\nabla_{\alpha} G\right)\left(s, r, \theta+\Psi_{j}\right)=\nabla_{\alpha}\left\{G\left(s, r, \theta+\Psi_{j}\right)\right\}-\left(\partial_{\theta} G\right)\left(s, r, \theta+\Psi_{j}\right) \nabla_{\alpha} \Psi_{j}
$$

Substituting (5.6) into (5.5) and integrating by parts give

$$
\begin{aligned}
I_{w h i t e}\left(\partial_{\mu} F\right)= & \sum_{j, \alpha=0}^{1}\left\{\iint_{\partial(\text { white })} d \sigma \int_{0}^{1} r K_{2} a_{\mu}^{\alpha}\left(\theta+\Psi_{j}\right) n_{\alpha} G\left(s, r, \theta+\Psi_{j}\right) d \tau\right. \\
& -\iint_{w h i t e} d r d s \int_{0}^{1} \nabla_{\alpha}\left\{r K_{2} a_{\mu}^{\alpha}\left(\theta+\Psi_{j}\right)\right\} G\left(s, r, \theta+\Psi_{j}\right) d \tau \\
& \left.-\iint_{w h i t e} r d r d s \int_{0}^{1} K_{2} a_{\mu}^{\alpha}\left(\theta+\Psi_{j}\right)\left(\partial_{\theta} G\right)\left(s, r, \theta+\Psi_{j}\right) \nabla_{\alpha} \Psi_{j} d \tau\right\} \\
+ & \iint_{w h i t e} d r d s \int_{-\varphi}^{\varphi} K_{1} a_{\mu}^{2}(\theta+\psi)\left(\partial_{\theta} G\right)(s, r, \theta+\psi) d \psi
\end{aligned}
$$


Similarly,

$$
\begin{aligned}
I_{r e d}\left(\partial_{\mu} F\right)= & \sum_{\alpha=0}^{1} \iint_{r e d} r d r d s \int_{-\pi}^{\pi} K_{1} a_{\mu}^{\alpha}(\theta+\psi)\left(\nabla_{\alpha} G\right)(s, r, \theta+\psi) d \psi \\
& +\iint_{r e d} d r d s \int_{-\pi}^{\pi} K_{1} a_{\mu}^{2}(\theta+\psi)\left(\partial_{\theta} G\right)(s, r, \theta+\psi) d \psi \\
= & \sum_{\alpha=0}^{1} \iint_{\partial(r e d)} r d \sigma \int_{-\pi}^{\pi} K_{1} a_{\mu}^{\alpha}(\theta+\psi) n_{\alpha} G(s, r, \theta+\psi) d \psi \\
& -\sum_{\alpha=0}^{1} \iint_{r e d} d r d s \int_{-\pi}^{\pi} \nabla_{\alpha}\left\{r K_{1}\right\} a_{\mu}^{\alpha}(\theta+\psi) G(s, r, \theta+\psi) d \psi \\
& +\iint_{r e d} d r d s \int_{-\pi}^{\pi} K_{1} a_{\mu}^{2}(\theta+\psi)\left(\partial_{\theta} G\right)(s, r, \theta+\psi) d \psi
\end{aligned}
$$

Thus we get the representation formula from (5.7) and (5.8).

The following proposition is used to estimate the terms appearing in Proposition 5.2. This was shown in M. Kovalyov[10], except the estimates containing the derivatives of $\Psi$. For the sake of completeness, we give the proof of all.

\section{Proposition 5.3}

I. Let $(s, r) \in D^{\prime}$. Then the following estimates hold:

$$
\begin{aligned}
& \text { (i) } \int_{-\varphi}^{\varphi} K_{1} d \psi=2 \int_{0}^{1} K_{2} d \tau \leq \frac{M}{(a r)^{1 / 2}} \log \left[2+\frac{a r}{\left(r-r_{1}\right)\left(r+r_{2}\right)} \chi(t-s-a)\right] \\
& \text { (ii) } \int_{0}^{1}\left\{\left|\partial_{s} K_{2}\right|+\left|\partial_{r} K_{2}\right|\right\} d \tau \leq \frac{M}{(a r)^{1 / 2}(r+s+a-t)} \\
& \text { (iii) } \int_{0}^{1} K_{2}\left\{\left|\partial_{s} \Psi\right|+\left|\partial_{r} \Psi\right|\right\} d \tau \leq \frac{M(a+r)}{\left\{a r\left(r^{2}-r_{1}^{2}\right)\left(r_{2}^{2}-r^{2}\right)\right\}^{1 / 2}}
\end{aligned}
$$

II. Let $(s, r) \in D^{\prime \prime}$. Then the following estimates hold:

$$
\begin{aligned}
& \text { (i) } \int_{-\pi}^{\pi} K_{1} d \psi \leq \frac{M}{\left\{\left(r+r_{1}\right)\left(r_{2}-r\right)\right\}^{1 / 2}} \log \left[2+\frac{a r}{\left(r_{1}-r\right)\left(r_{2}+r\right)}\right] \\
& \text { (ii) } \int_{-\pi}^{\pi}\left\{\left|\partial_{s} K_{1}\right|+\left|\partial_{r} K_{1}\right|\right\} d \psi \leq \frac{M}{\left(r_{1}-r\right)\left\{\left(r+r_{1}\right)\left(r_{2}-r\right)\right\}^{1 / 2}}
\end{aligned}
$$

Proof. The following identity can be easily verified by simple computation.

$$
1+P=\frac{\left(r+r_{2}\right)(r+a-t+s)}{2 a r}, 1-P=\frac{\left(r_{2}-r\right)(t-s-a+r)}{2 a r}
$$


I-(i). Changing variable by the map $\psi=\Psi$, we have

$$
\begin{aligned}
\int_{-\varphi}^{\varphi} K_{1} d \psi & =2 \int_{0}^{1} K_{2} d \tau \\
& =\frac{2^{1 / 2}}{(a r)^{1 / 2}} \int_{0}^{1}\{\tau(1-\tau)(2+P \tau-\tau)\}^{-1 / 2} d \tau
\end{aligned}
$$

First, we notice that in the domain $D^{\prime}$,

$$
\begin{aligned}
& |P|<1 \\
& 2+P \tau-\tau=(P+1) \tau+2(1-\tau) \geq 2(1-\tau) \text { for } \tau>0
\end{aligned}
$$

Thus, splitting the interval of integration into two pieces, we have

$$
\begin{aligned}
\int_{0}^{1 / 2}\{ & \tau(1-\tau)(2+P \tau-\tau)\}^{-1 / 2} d \tau \leq 2^{1 / 2} \int_{0}^{1 / 2} \tau^{-1 / 2} d \tau=2 \\
\int_{1 / 2}^{1}\{ & \\
& \leq 2^{1 / 2} \int_{1 / 2}^{1}\{(1-\tau)(2+P \tau-\tau)\}^{-1 / 2} d \tau \\
& \leq 2^{1 / 2} \int_{0}^{1} \partial_{\tau}\left\{-2(1-\tau)^{1 / 2}\right\}(2+P \tau-\tau)^{-1 / 2} d \tau \\
& =2+2^{1 / 2}(1-P) \int_{0}^{1}(1-\tau)^{1 / 2}(2+P \tau-\tau)^{-3 / 2} d \tau \\
& \leq 2+(1-P) \int_{0}^{1}(2+P \tau-\tau)^{-1} d \tau \\
& \leq M \log \left[2+\frac{1}{1+P}\right]
\end{aligned}
$$

Since $P+1>1 / 2$ for $t-s \leq a$, the estimate I-(i) follows from (5.10)-(5.12) and (5.9).

I-(ii). Since

$$
\begin{aligned}
& \partial_{s} K_{2}=\frac{-(t-s) \tau^{1 / 2}}{(2 a r)^{3 / 2}(1-\tau)^{1 / 2}(2+P \tau-\tau)^{3 / 2}} \\
& \partial_{r} \cdot K_{2}=-\frac{1}{2 r} K_{2}-\frac{\tau^{1 / 2}}{2^{3 / 2}(a r)^{1 / 2}(1-\tau)^{1 / 2}(2+P \tau-\tau)^{3 / 2}}\left(\frac{1}{a}-\frac{P}{r}\right)
\end{aligned}
$$

then we have

$$
\begin{aligned}
& \int_{0}^{1}\left\{\left|\partial_{s} K_{2}\right|+\left|\partial_{r} K_{2}\right|\right\} d \tau \\
& \quad \leq \frac{1}{2 r} \int_{0}^{1} K_{2} d \tau+\frac{t-s+a+r}{(2 a r)^{3 / 2}} \int_{0}^{1} \frac{\tau^{1 / 2}}{(1-\tau)^{1 / 2}(2+P \tau-\tau)^{3 / 2}} d \tau
\end{aligned}
$$


By I-(i), we have

$$
\begin{aligned}
\frac{1}{2 r} \int_{0}^{1} K_{2} d \tau & \leq M a(a r)^{-3 / 2} \log \left[2+(1+P)^{-1}\right] \\
& \leq M a(a r)^{-3 / 2}(1+P)^{-1}
\end{aligned}
$$

On the other hand, since

$$
\int_{0}^{1} \frac{\tau^{1 / 2}}{(1-\tau)^{1 / 2}(2+P \tau-\tau)^{3 / 2}} d \tau \leq \int_{0}^{1}(1-\tau)^{-1 / 2}(2+P \tau-\tau)^{-3 / 2} d \tau
$$

we have by the method from which (5.12) was derived,

$$
\int_{0}^{1} \frac{\tau^{1 / 2}}{(1-\tau)^{1 / 2}(2+P \tau-\tau)^{3 / 2}} d \tau \leq \frac{3}{2^{1 / 2}} \frac{1}{1+P}
$$

Therefore it follows from $(5.13),(5.14)$ and (5.15) that

$$
\begin{aligned}
\int_{0}^{1}\left\{\left|\partial_{s} K_{2}\right|+\left|\partial_{r} K_{2}\right|\right\} d \tau & \leq \frac{M(t-s+a+r)}{(a r)^{3 / 2}} \frac{1}{1+P} \\
& \leq \frac{M}{(a r)^{1 / 2}(r+s+a-t)}
\end{aligned}
$$

I-(iii). We can easily verify that

$$
\begin{aligned}
\partial_{r} \Psi & =\left(\frac{P}{r}-\frac{1}{a}\right) \frac{\tau^{1 / 2}}{\{(1-P)(2+P \tau-\tau)\}^{1 / 2}} \\
\partial_{s} \Psi & =-\frac{t-s}{a r} \frac{\tau^{1 / 2}}{\{(1-P)(2+P \tau-\tau)\}^{1 / 2}} .
\end{aligned}
$$

We use the same method as we used in I-(i) and obtain

$$
\begin{aligned}
\int_{0}^{1} K_{2}\left\{\left|\partial_{r} \Psi\right|+\left|\partial_{s} \Psi\right|\right\} d \tau \\
\quad=\frac{1}{(2 a r)^{1 / 2}}\left\{\left|\frac{1}{a}-\frac{P}{r}\right|+\frac{t-s}{a r}\right\} \frac{1}{(1-P)^{1 / 2}} \int_{0}^{1} \frac{d \tau}{(1-\tau)^{1 / 2}(2+P \tau-\tau)} \\
\quad \leq \frac{M}{(a r)^{1 / 2}} \frac{a+r}{a r} \frac{1}{\{(1-P)(1+P)\}^{1 / 2}} .
\end{aligned}
$$

Thus we get the estimate I-(iii). 
II-(i). In the domain $D^{\prime \prime}, P<-1$ and $t-s>a+r$. Therefore,

$$
\begin{aligned}
\int_{-\pi}^{\pi} K_{1} d \psi & =\frac{2}{\left\{(t-s)^{2}-a^{2}-r^{2}\right\}^{1 / 2}} \int_{0}^{\pi} \frac{d \psi}{\left(1-P^{-1} \cos \psi\right)^{1 / 2}} \\
5.16) & \leq \frac{2}{\left\{(t-s)^{2}-a^{2}-r^{2}\right\}^{1 / 2}}\left\{\int_{0}^{3 \pi / 4} \frac{d \psi}{\left(1-2^{-1 / 2}\right)^{1 / 2}}+\int_{3 \pi / 4}^{\pi} \frac{d \psi}{\left(1-P^{-1} \cos \psi\right)^{1 / 2}}\right\}
\end{aligned}
$$

Further,

$$
\begin{aligned}
\int_{3 \pi / 4}^{\pi} \frac{d \psi}{\left(1-P^{-1} \cos \psi\right)^{1 / 2}} & =\int_{0}^{\pi / 4} \frac{d \psi}{\left(1+P^{-1} \cos \psi\right)^{1 / 2}} \\
& =\int_{0}^{\pi / 4} \frac{d \psi}{\left\{1-\cos \psi+\left(1+P^{-1}\right) \cos \psi\right\}^{1 / 2}} \\
& \leq \int_{0}^{\pi / 4} \frac{d \psi}{\left\{2 \pi^{-2} \psi^{2}+\left(1+P^{-1}\right) 2^{-1 / 2}\right\}^{1 / 2}} \\
& \leq M\left\{1-\log \left(1+P^{-1}\right)\right\} \\
& =M\left\{1+\log \left(1-\frac{1}{1+P}\right)\right\}
\end{aligned}
$$

From (5.17), (5.9) and

$$
(t-s)^{2}-a^{2}-r^{2}=-2 a r P \geq \operatorname{ar}(1-P)=\frac{1}{2}\left(r+r_{1}\right)\left(r_{2}-r\right),
$$

we get II-(i).

II-(ii). We can easily see that

$$
\begin{aligned}
& \partial_{s} K_{1}=\frac{t-s}{\left((t-s)^{2}-a^{2}-r^{2}+2 a r \cos \psi\right)^{3 / 2}} \\
& \partial_{r} K_{1}=\frac{r-a \cos \psi}{\left((t-s)^{2}-a^{2}-r^{2}+2 a r \cos \psi\right)^{3 / 2}}
\end{aligned}
$$

Thus,

$$
\begin{aligned}
\int_{-\pi}^{\pi}\left\{\left|\partial_{s} K_{1}\right|+\left|\partial_{r} K_{1}\right|\right\} d \psi & \leq 2(t-s) \int_{-\pi}^{\pi} \frac{d \psi}{\left((t-s)^{2}-a^{2}-r^{2}+2 a r \cos \psi\right)^{3 / 2}} \\
& =\frac{4(t-s)}{(-2 a r P)^{3 / 2}} \int_{0}^{\pi} \frac{d \psi}{\left(1-P^{-1} \cos \psi\right)^{3 / 2}} .
\end{aligned}
$$

We get by the same way as the proof of II-(i) that

$$
\begin{aligned}
\int_{0}^{\pi} \frac{d \psi}{\left(1-P^{-1} \cos \psi\right)^{3 / 2}} & \leq \int_{0}^{4 \pi / 3} \frac{d \psi}{\left(1-2^{-1 / 2}\right)^{3 / 2}}+\int_{0}^{\pi / 4} \frac{d \psi}{\left\{2 \pi^{-2} \psi^{2}+\left(1+P^{-1}\right) 2^{-1 / 2}\right\}^{3 / 2}} \\
& \leq M\left(1+\frac{P}{1+P}\right) \\
& \leq M \frac{P}{1+P}
\end{aligned}
$$


Therefore it follows from (5.18) and (5.19) that

$$
\begin{aligned}
\int_{-\pi}^{\pi}\left\{\left|\partial_{s} K_{1}\right|+\left|\partial_{r} K_{1}\right|\right\} d \psi & \leq \frac{M(t-s)}{(-2 a r P)^{3 / 2}} \frac{P}{1+P} \\
& \leq \frac{M(t-s)}{(a r)^{3 / 2}(1-P)^{1 / 2}} \frac{-1}{1+P} \\
& \leq \frac{M}{\left(r_{1}-r\right)(a r)^{1 / 2}(1-P)^{1 / 2}}
\end{aligned}
$$

and we get the estimate II-(ii).

Now we can show the estimates for the first derivatives of the solution to the initial value problem (5.1).

Proposition 5.4 Let $u \in C^{\infty}\left([0, T) \times \mathbf{R}^{2}\right)$ be the solution of the initial value problem (5.1). And let $w(s, r)$ be a positive function that satisfies

$$
\begin{aligned}
& \text { (i) } k-1 \leq r \leq k+1 \Rightarrow \frac{1}{M} w(s, k) \leq w(s, r) \leq M w(s, k) \\
& (k=1,2, \cdots ; M \text { is independent of } s, k, r) \\
& \frac{1}{w(s, r)} \leq M\left\{\sum_{i=1}^{L} \frac{1}{(r+s+1)\left(\left|r-c_{i} s\right|+1\right)}+\frac{1}{(r+s+1)^{1+2 \gamma}(r+1)^{1-2 \gamma}}\right. \\
& \left.+\frac{1}{(r+s+1)^{1+\epsilon}(|r-s|+1)^{1-\epsilon}}\right\} \\
& \left(c_{i} \neq 1(i=1,2, \cdots, L), 0<\gamma<1 / 2,0<\epsilon<1\right)
\end{aligned}
$$

Then the following estimate holds:

$$
\begin{aligned}
|\partial u(t, x)| \leq & \frac{M}{(|x|+1)^{1 / 2-\gamma}(|x|+t+1)^{\gamma}(|| x|-t|+1)^{1 / 2}}\left\{\sum_{|A| \leq 3} \sup _{0<s<t}\left\|w(s,|\cdot|) \mathcal{D}^{A} F(s, \cdot)\right\|_{0}\right. \\
(5.22) & \left.+\sum_{|A| \leq 2} \sup _{0<s<t}\left\|w(s,|\cdot|) \mathcal{D}^{A} \partial_{t} F(s, \cdot)\right\|_{0}\right\} .
\end{aligned}
$$

Proof. By (5.4) and Proposition 5.2, we have

$$
\begin{aligned}
|\partial u(t, x)| \leq & \left\{\sup _{0<s<t} \sup _{x \in \mathbf{R}^{2}}|x|^{1 / 2} w(t,|x|)|F(t, x)|+\sup _{0<s<t} \sup _{x \in \mathbf{R}^{2}}|x|^{1 / 2} w(t,|x|)|\partial F(t, x)|\right. \\
& \left.+\sup _{0<s<t} \sup _{x \in \mathbf{R}^{2}}|x|^{1 / 2} w(t,|x|)|\Omega F(t, x)|\right\}\left\{I_{1}^{\prime}+\cdots+I_{5}^{\prime}+I_{1}^{\prime \prime}+\cdots+I_{4}^{\prime \prime}\right\}
\end{aligned}
$$


where $I_{i}^{\prime}(i=1, \ldots, 5)$ and $I_{i}^{\prime \prime}(i=1, \ldots, 4)$ are defined as follows.

$$
\begin{aligned}
I_{1}^{\prime} & =\iint_{\text {blue }} \frac{r^{1 / 2}}{w(s, r)} d r d s \int_{-\varphi}^{\varphi} K_{1} d \psi \\
I_{2}^{\prime} & =\iint_{\partial(\text { white })} \frac{r^{1 / 2}}{w(s, r)} d \sigma \int_{0}^{1} K_{2} d \tau \\
I_{3}^{\prime} & =\iint_{\text {white }} \frac{1}{r^{1 / 2} w(s, r)} d r d s \int_{0}^{1} K_{2} d \tau \\
I_{4}^{\prime} & =\iint_{\text {white }} \frac{r^{1 / 2}}{w(s, r)} d r d s \int_{0}^{1}\left\{\left|\partial_{s} K_{2}\right|+\left|\partial_{r} K_{2}\right|\right\} d \tau \\
I_{5}^{\prime} & =\iint_{w h i t e} \frac{r^{1 / 2}}{w(s, r)} d r d s \int_{0}^{1} K_{2}\left\{\left|\partial_{s} \Psi\right|+\left|\partial_{r} \Psi\right|\right\} d \tau \\
I_{1}^{\prime \prime} & =\iint_{\text {black }} \frac{r^{1 / 2}}{w(s, r)} d r d s \int_{-\pi}^{\pi} K_{1} d \psi \\
I_{2}^{\prime \prime} & =\iint_{\partial(\text { red })} \frac{r^{1 / 2}}{w(s, r)} d \sigma \int_{-\pi}^{\pi} K_{1} d \psi \\
I_{3}^{\prime \prime} & =\iint_{\text {red }} \frac{1}{r^{1 / 2} w(s, r)} d r d s \int_{-\pi}^{\pi} K_{1} d \psi \\
I_{4}^{\prime \prime} & =\iint_{\text {red }} \frac{r^{1 / 2}}{w(s, r)} d r d s \int_{-\pi}^{\pi}\left\{\left|\partial_{s} K_{1}\right|+\left|\partial_{r} K_{1}\right|\right\} d \psi
\end{aligned}
$$

Here, $I_{i}^{\prime}(i=1, \ldots, 5)$ are integrals that are related to the domain $D^{\prime}$, and $I_{i}^{\prime \prime}(i=1, \ldots, 4)$ to the domain $D^{\prime \prime}$. We show in the following that

$$
\begin{aligned}
I_{i}^{\prime} & \leq \frac{M}{(a+1)^{1 / 2-\gamma}(a+t+1)^{\gamma}(|a-t|+1)^{1 / 2}} \quad(i=1, \ldots, 5), \\
I_{i}^{\prime \prime} & \leq \frac{M}{(a+1)^{1 / 2-\gamma}(a+t+1)^{\gamma}(|a-t|+1)^{1 / 2}} \quad(i=1, \ldots, 4),
\end{aligned}
$$

where $a=|x|$. By Lemma 6 in [10], it holds that

$$
\left|x\left\|f(x) \mid w(s,|x|)^{2} \leq M \sum_{|A| \leq 2}\right\| w(s,|\cdot|) \mathcal{D}^{A} f \|_{0}^{2}\right.
$$

for $f \in C_{0}^{\infty}\left(\mathbf{R}^{2}\right)$. Then we get the estimate (5.22) from (5.23), (5.24), (5.25) and (5.26).

First, we prove (5.24). To prove this, we introduce some notations. Set

$$
\begin{aligned}
\xi(s, r) & =\xi_{1}(s, r)+\xi_{2}(s, r) \\
\xi_{1}(s, r) & =\sum_{i=1}^{L} \frac{1}{(r+s+1)\left(\left|r-c_{i} s\right|+1\right)}+\frac{1}{(r+s+1)^{1+2 \gamma}(r+1)^{1-2 \gamma}} \\
\xi_{2}(s, r) & =\frac{1}{(r+s+1)^{1+\epsilon}(|r-s|+1)^{1-\epsilon}}
\end{aligned}
$$


Then by the assumption (5.21) on $w(s, r)$,

$$
\frac{1}{w(s, r)} \leq M \xi(s, r)
$$

Moreover, set

$$
\begin{aligned}
\eta(s, r) & =\eta_{1}(s, r)+\eta_{2}(s, r) \\
\eta_{1}(s, r) & =\sum_{i=1}^{L} \frac{1}{(r+s+1)^{\tilde{\lambda}}\left(\left|r-c_{i} s\right|+1\right)}+\frac{1}{(r+s+1)^{\tilde{\lambda}+2 \gamma}(r+1)^{1-2 \gamma}} \\
\eta_{2}(s, r) & =\frac{1}{(r+s+1)^{\tilde{\lambda}+\epsilon}(|r-s|+1)^{1-\epsilon}}
\end{aligned}
$$

where

$$
0<\lambda<\min \{\gamma, 1 / 2-\gamma\}, \tilde{\lambda}=1 / 2-\gamma-\lambda
$$

Since $r+s \geq|a-t|$ for $(s, r) \in D^{\prime}$, we have

$$
\begin{aligned}
\xi_{i}(s, r) & \leq \frac{M}{(|a-t|+1)^{1 / 2+\gamma+\lambda}} \eta_{i}(s, r) \quad(i=1,2) \\
\frac{1}{w(s, r)} & \leq \frac{M}{(|a-t|+1)^{1 / 2+\gamma+\lambda}} \eta(s, r)
\end{aligned}
$$

for $(s, r) \in D^{\prime}$. But in the estimate of $I_{1}^{\prime}$ and $I_{5}^{\prime}, \xi_{2}(s, r)$ is treated in another way.

(i) Estimate of $I_{1}^{\prime}$

By Proposition 5.3.I.(i),

$$
I_{1}^{\prime} \leq \frac{M}{a^{1 / 2}} \iint_{\text {blue }} \frac{1}{w(s, r)} \log \left[2+\frac{a r}{\left(r-r_{1}\right)\left(r+r_{2}\right)} \chi(t-s-a)\right] d r d s
$$

Therefore it follows from (5.31) and (5.20) that

$$
\begin{aligned}
I_{1}^{\prime} \leq & \frac{M}{a^{1 / 2}}\left\{\int_{0}^{t} \frac{d s}{w\left(s, r_{1}\right)} \int_{r_{1}}^{r_{1}+\delta} \log \left[2+\frac{a r}{\left(r-r_{1}\right)\left(r+r_{2}\right)} \chi(t-s-a)\right] d r\right. \\
& \left.+\int_{0}^{t} \frac{d s}{w\left(s, r_{2}\right)} \int_{r_{2}-\delta}^{r_{2}} \log \left[2+\frac{a r}{\left(r-r_{1}\right)\left(r+r_{2}\right)} \chi(t-s-a)\right] d r\right\}
\end{aligned}
$$

Let us consider the integrals of $\log \left[2+a r /\left(r-r_{1}\right)\left(r+r_{2}\right) \cdot \chi(t-s-a)\right]$. For $0<s<t-a$ and $r_{2}-\delta<r<r_{2}$, it follows from (5.2) and (5.3) that

$$
r-r_{1}>r_{2}-\delta-r_{1}=2 a-\delta>a
$$


Then we have

$$
\log \left[2+\frac{a r}{\left(r-r_{1}\right)\left(r+r_{2}\right)}\right] \leq \log \left[2+\frac{a}{r-r_{1}}\right] \leq \log 3 .
$$

For $0<s<t-a$ and $r_{1}<r<r_{1}+\delta$, we have

$$
\begin{aligned}
\int_{r_{1}}^{r_{1}+\delta} \log \left[2+\frac{a r}{\left(r-r_{1}\right)\left(r+r_{2}\right)}\right] d r & \leq \int_{r_{1}}^{r_{1}+\delta} \log \left[2+\frac{r}{r-r_{1}}\right] d r \\
& =\delta\left[\left\{\log \left(3 \delta+r_{1}\right)-\log \delta\right\}+\frac{r_{1}}{3 \delta} \log \left(1+3 \delta / r_{1}\right)\right] \\
& \leq \delta \log (3 / 2+t-a)+\delta^{1 / 2} 2 e^{-1}+\delta \\
& \leq M \delta^{1 / 2} \log [2+|a-t|] .
\end{aligned}
$$

Therefore it follows from (5.32), (5.33) and (5.34) that

$$
I_{1}^{\prime} \leq \frac{M \delta^{1 / 2}}{a^{1 / 2}} \log [2+|a-t|]\left\{\int_{0}^{t} \frac{d s}{w\left(s, r_{1}\right)}+\int_{0}^{t} \frac{d s}{w\left(s, r_{2}\right)}\right\} .
$$

We next show

$$
\int_{0}^{t} \frac{d s}{w\left(s, r_{i}\right)} \leq \frac{M}{(|a-t|+1)^{1 / 2+\gamma+\lambda}} \quad(i=1,2) .
$$

We use (5.30) for $1 / w\left(s, r_{2}\right)$ and obtain

$$
\int_{0}^{t} \frac{d s}{w\left(s, r_{2}\right)} \leq \frac{M}{(|a-t|+1)^{1 / 2+\gamma+\lambda}} \int_{0}^{t} \eta\left(s, r_{2}\right) d s .
$$

Moreover,

$$
\begin{aligned}
& \int_{0}^{t} \eta\left(s, r_{2}\right) d s \\
& \quad \leq M \int_{0}^{t}\left\{\sum_{i=1}^{L} \frac{1}{\left(\left|r_{2}-c_{i} s\right|+1\right)^{1+\tilde{\lambda}}}+\frac{1}{\left(\left|r_{2}-s\right|+1\right)^{1+\tilde{\lambda}}}+\frac{1}{\left(r_{2}+1\right)^{1+\tilde{\lambda}}}\right\} d s \\
& \quad \leq M \int_{-\infty}^{\infty}\left\{\sum_{i=1}^{L} \frac{1}{\left(\left|r_{2}-c_{i} s\right|+1\right)^{1+\tilde{\lambda}}}+\frac{1}{\left(\left|r_{2}-s\right|+1\right)^{1+\tilde{\lambda}}}+\frac{1}{\left(\left|r_{2}\right|+1\right)^{1+\tilde{\lambda}}}\right\} d s \leq M .
\end{aligned}
$$

Therefore, from (5.37) and (5.38) we have (5.36) for $i=2$. The treatment for $i=1$ is slightly different. We remark that $\left|r_{1}-s\right|=|a-t|$ for $(t-a)_{+}<s<t$ by the definition (5.2), where $x_{+}=\max \{0, x\}$. Then we see from (5.28) that

$$
\xi_{2}\left(s, r_{1}\right) \leq \frac{1}{(|a-t|+1)^{1 / 2+\gamma+\lambda}\left(r_{1}+s+1\right)^{1+\min \{\epsilon, \bar{\lambda}\}}}
$$


for $(t-a)_{+}<s<t$. Therefore it follows (5.27), (5.29) and (5.39) that

$$
\begin{aligned}
\int_{0}^{t} \frac{d s}{w\left(s, r_{1}\right)} \leq & \frac{M}{(|a-t|+1)^{1 / 2+\gamma+\lambda}}\left\{\int_{0}^{t} \eta_{1}\left(s, r_{1}\right) d s+\int_{0}^{(t-a)_{+}} \eta_{2}\left(s, r_{1}\right) d s\right. \\
& \left.+\int_{(t-a)_{+}}^{t} \frac{d s}{\left(r_{1}+s+1\right)^{1+\min \{\epsilon, \dot{\lambda}\}}}\right\} \\
\leq & \frac{M}{(|a-t|+1)^{1 / 2+\gamma+\lambda}} .
\end{aligned}
$$

Combining (5.35) and (5.36), we have

$$
\begin{aligned}
I_{1}^{\prime} & \leq \frac{M \delta^{1 / 2}}{a^{1 / 2}(|a-t|+1)^{1 / 2+\gamma+\lambda}} \log [2+|a-t|] \\
& \leq \frac{M}{(a+1)^{1 / 2}(|a-t|+1)^{1 / 2+\gamma}} \\
& \leq \frac{M}{(a+1)^{1 / 2-\gamma}(a+t+1)^{\gamma}(|a-t|+1)^{1 / 2}} .
\end{aligned}
$$

Here we use the fact that

$$
\frac{a+t+1}{(a+1)(|a-t|+1)} \leq 4 \text { for } a, t \geq 0
$$

(ii) Estimate of $I_{2}^{\prime}$

By Proposition 5.3.I.(i),

$$
I_{2}^{\prime} \leq \frac{M}{a^{1 / 2}} \iint_{\partial(\text { white })} \frac{1}{w(s, r)} \log \left[2+\frac{a r}{\left(r-r_{1}\right)\left(r+r_{2}\right)} \chi(t-s-a)\right] d \sigma .
$$

Let $0 \leq s \leq t-a$ and $(s, r) \in \overline{w h i t e}$. Then

$$
\frac{a r}{\left(r-r_{1}\right)\left(r+r_{2}\right)} \leq \frac{r}{r-r_{1}} \leq \frac{r_{1}+1 / 2}{1 / 2} \leq 2(t-a+1 / 2) .
$$

So we have

$$
\frac{a r}{\left(r-r_{1}\right)\left(r_{2}+r\right)} \chi(t-s-a) \leq 2(2+|a-t|)
$$

for $(s, r) \in \overline{\text { white. }}$. Hence from (5.42) and (5.43) we get

$$
I_{2}^{\prime} \leq \frac{M}{a^{1 / 2}} \log [2+|a-t|] \iint_{\partial(w h i t e)} \frac{d \sigma}{w(s, r)} .
$$


We have already computed the integral of $1 / w(s, r)$ in the estimate of $I_{1}^{\prime}$, except the one on $\{0\} \times(|a-t|+\delta, a+t-\delta)$. Applying (5.30) for $s=0$, we have

$$
\int_{|a-t|+\delta}^{a+t-\delta} \frac{d r}{w(0, r)} \leq \frac{M}{(|a-t|+1)^{1 / 2+\gamma+\lambda}} .
$$

Therefore it follows from $(5.44),(5.36)$ and (5.45) that

$$
I_{2}^{\prime} \leq \frac{M}{a^{1 / 2}(|a-t|+1)^{1 / 2+\gamma+\lambda}} \log [2+|a-t|] .
$$

Since $a \geq \delta=1 / 2$ when the domain white is not empty, we have (5.24) for $i=2$ by the way from which (5.40) was derived.

(iii) Estimate of $I_{3}^{\prime}$

By Proposition 5.3.I.(i),

$$
I_{3}^{\prime} \leq \frac{M}{a^{1 / 2}} \iint_{w h i t e} \frac{1}{r w(s, r)} \log \left[2+\frac{a r}{\left(r-r_{1}\right)\left(r+r_{2}\right)} \chi(t-s-a)\right] d r d s .
$$

Further, by (5.30) and (5.43),

$$
I_{3}^{\prime} \leq \frac{M}{a^{1 / 2}(|a-t|+1)^{1 / 2+\gamma+\lambda}} \log [2+|a-t|] \iint_{w h i t e} \frac{\eta(s, r)}{r} d r d s .
$$

Since $r \geq \delta=1 / 2$ in the domain white, we have

$$
\begin{aligned}
\frac{\eta(s, r)}{r} \leq & M\left\{\sum_{i=1}^{L} \frac{1}{(r+1)^{1+\tilde{\lambda} / 2}\left(\left|r-c_{i} s\right|+1\right)^{1+\tilde{\lambda} / 2}}\right. \\
& \left.+\frac{1}{(r+1)^{2+\tilde{\lambda}}}+\frac{1}{(r+1)^{1+\tilde{\lambda} / 2}(|r-s|+1)^{1+\tilde{\lambda} / 2}}\right\}
\end{aligned}
$$

for $(s, r) \in$ white. Concerning the right-hand side of (5.49), the integral of the first and the third term are shown to be bounded by a constand $M$ in the same way as (5.38). As for the second term, we see that

$$
\begin{aligned}
\iint_{\text {white }} \frac{d r d s}{(r+1)^{2+\tilde{\lambda}}} & \leq \int_{0}^{t} d s \int_{r_{1}}^{r_{2}} \frac{d r}{(r+1)^{2+\tilde{\lambda}}} \\
& \leq \frac{1}{1+\tilde{\lambda}} \int_{0}^{t} \frac{d s}{\left(r_{1}+1\right)^{1+\tilde{\lambda}}} \leq M
\end{aligned}
$$

Therefore it follows from (5.49) and (5.50) that

$$
\iint_{w h i t e} \frac{\eta(s, r)}{r} d r d s \leq M
$$


Hence from (5.48) and (5.51) we have (5.24) for $i=3$.

(iv) Estimate of $I_{4}^{\prime}$

By Proposition 5.3.I.(ii),

$$
I_{4}^{\prime} \leq \frac{M}{a^{1 / 2}} \iint_{w h i t e} \frac{1}{w(s, r)(r+s+a-t)} d r d s .
$$

Applying (5.30) to the right-hand side of (5.52), we obtain

$$
I_{4}^{\prime} \leq \frac{M}{a^{1 / 2}(|a-t|+1)^{1 / 2+\gamma+\lambda}} \iint_{w h i t e} \frac{\eta(s, r)}{r+s+a-t} d r d s .
$$

Since $r+s+a-t \geq \delta=1 / 2$ in the domain white, we have

$$
\begin{aligned}
\frac{\eta(s, r)}{r+s+a-t} \leq & \frac{M}{r+s+a-t+1}\left\{\sum_{i=1}^{L} \frac{1}{(r+s+1)^{\tilde{\lambda} / 2}\left(\left|r-c_{i} s\right|+1\right)^{1+\tilde{\lambda} / 2}}\right. \\
& \left.+\frac{1}{(r+s+1)^{\tilde{\lambda} / 2}(r+1)^{1+\tilde{\lambda} / 2}}+\frac{1}{(r+s+1)^{\tilde{\lambda} / 2}(|r-s|+1)^{1+\tilde{\lambda} / 2}}\right\}
\end{aligned}
$$

for $(s, r) \in$ white. Hence we find by the change of variables $(\alpha, \beta)=(s+r, s-r)$ that

$$
\iint_{w h i t e} \frac{\eta(s, r)}{r+s+a-t} d r d s \leq M
$$

Therefore from (5.53), (5.54) and (5.41) we obtain (5.24) for $i=4$.

(v) Estimate of $I_{5}^{\prime}$

By Proposition 5.3.I.(iii),

$$
I_{5}^{\prime} \leq \frac{M}{a^{1 / 2}} \iint_{w h i t e} \frac{1}{w(s, r)} \frac{a+r}{\left\{\left(r^{2}-r_{1}^{2}\right)\left(r_{2}^{2}-r^{2}\right)\right\}^{1 / 2}} d r d s
$$

We notice that for $(s, r) \in$ white,

$$
\begin{aligned}
& r_{2}+r \geq a, r_{2}+r \geq r ; \\
& r+r_{1} \geq a, r+r_{1} \geq r \quad \text { for } r \geq\left(r_{2}-r_{1}\right) / 2 ; \\
& r_{2}-r \geq a, r_{2}-r \geq r \quad \text { for } \quad r \leq\left(r_{2}-r_{1}\right) / 2 .
\end{aligned}
$$

Hence we have

$$
\frac{a+r}{\left\{\left(r^{2}-r_{1}^{2}\right)\left(r_{2}^{2}-r^{2}\right)\right\}^{1 / 2}} \leq 2\left\{\frac{1}{\left(r^{2}-r_{1}^{2}\right)^{1 / 2}}+\frac{1}{\left\{\left(r-r_{1}\right)\left(r_{2}-r\right)\right\}^{1 / 2}}\right\}
$$


for $(s, r) \in$ white. Therefore it follows from (5.55), (5.27) and (5.56) that

$$
I_{5}^{\prime} \leq \frac{M}{a^{1 / 2}} \iint_{w h i t e} \xi(s, r)\left\{\frac{1}{\left(r^{2}-r_{1}^{2}\right)^{1 / 2}}+\frac{1}{\left\{\left(r-r_{1}\right)\left(r_{2}-r\right)\right\}^{1 / 2}}\right\} d r d s .
$$

We show in the following that

$$
\iint_{w h i t e} \xi(s, r) \frac{1}{\left(r^{2}-r_{1}^{2}\right)^{1 / 2}} d r d s \leq \frac{M}{(|a-t|+1)^{1 / 2+\gamma+\lambda}}
$$

We use (5.29) for $\xi_{1}(s, r)$ and obtain

$$
\begin{aligned}
& \iint_{\text {white }} \xi_{1}(s, r) \frac{1}{\left(r^{2}-r_{1}^{2}\right)^{1 / 2}} d r d s \\
& \quad \leq \frac{M}{(|a-t|+1)^{1 / 2+\gamma+\lambda}} \iint_{w h i t e} \eta_{1}(s, r) \frac{1}{\left(r^{2}-r_{1}^{2}\right)^{1 / 2}} d r d s .
\end{aligned}
$$

Since $r \pm(s+a-t) \geq \delta=1 / 2$ for $(s, r) \in$ white, we have

$$
\begin{aligned}
\frac{\eta_{1}(s, r)}{\left(r^{2}-r_{1}^{2}\right)^{1 / 2} \leq} & \frac{M}{(r+s+a-t+1)^{1 / 2}(r-s-a+t+1)^{1 / 2}} \eta_{1}(s, r) \\
\leq & \frac{M}{r+s+a-t+1}\left\{\sum_{i=1}^{L} \frac{1}{(r+s+1)^{\tilde{\lambda} / 2}\left(\left|r-c_{i} s\right|+1\right)^{1+\tilde{\lambda} / 2}}\right. \\
& \left.+\frac{1}{(r+s+1)^{\tilde{\lambda} / 2}(r+1)^{1+\tilde{\lambda} / 2}}\right\} \\
& +\frac{M}{r-s-a+t+1}\left\{\sum_{i=1}^{L} \frac{1}{(|r-s|+1)^{\tilde{\lambda} / 2}\left(\left|r-c_{i} s\right|+1\right)^{1+\tilde{\lambda} / 2}}\right. \\
& \left.+\frac{1}{(|r-s|+1)^{\tilde{\lambda} / 2}(r+1)^{1+\tilde{\lambda} / 2}}\right\}
\end{aligned}
$$

for $(s, r) \in$ white. Hence by the change of variables $(\alpha, \beta)=(s+r, s-r)$ we have

$$
\iint_{\text {white }} \frac{\eta_{1}(s, r)}{\left(r^{2}-r_{1}^{2}\right)^{1 / 2}} d r d s \leq M
$$

Therefore it follows from (5.59) and (5.60) that

$$
\iint_{\text {white }} \frac{\xi_{1}(s, r)}{\left(r^{2}-r_{1}^{2}\right)^{1 / 2}} d r d s \leq \frac{M}{(|a-t|+1)^{1 / 2+\gamma+\lambda}} .
$$

On the other hand, we see that, for $(s, r) \in$ white,

$$
\frac{\xi_{2}(s, r)}{\left(r^{2}-r_{1}^{2}\right)^{1 / 2}}
$$




$$
\begin{aligned}
\leq & \frac{M}{(r+s+a-t+1)^{1 / 2}(r-s-a+t+1)^{1 / 2}}\left[\{1-\chi(|r-s|-|a-t| / 2)\} \xi_{2}(s, r)\right. \\
& \left.+\chi(|r-s|-|a-t| / 2) \xi_{2}(s, r)\right] \\
\leq & \frac{M}{(|a-t|+1)^{1 / 2+\gamma+\lambda}} \frac{1}{(r+s+a-t+1)^{1 / 2}} \cdot \\
& \cdot\left\{\frac{1}{(r+s+1)^{1 / 2+\tilde{\lambda}+\epsilon}(|r-s|+1)^{1-\epsilon}}+\frac{1}{\left.(r-s-a+t+1)^{1 / 2}(r+s+1)^{1+\min \{\epsilon, \tilde{\lambda}\}}\right\}}\right. \\
\leq & \frac{1}{(|a-t|+1)^{1 / 2+\gamma+\lambda}} \frac{1}{(r+s+a-t+1)^{1 / 2}} \cdot \\
& \cdot\left\{\frac{1}{(r+s+1)^{1 / 2+\tilde{\lambda} / 2}(|r-s|+1)^{1+\tilde{\lambda} / 2}}\right. \\
& \left.+\frac{1}{(r-s-a+t+1)^{1 / 2}(|r-s|+1)^{1 / 2+\min \{\epsilon, \tilde{\lambda}\} / 2}(r+s+1)^{1 / 2+\min \{\epsilon, \tilde{\lambda}\} / 2}}\right\} .
\end{aligned}
$$

Here, we have used that $t-a>0$ on the support of $1-\chi(|r-s|-|a-t| / 2)$. Therefore it follows that

$$
\iint_{\text {white }} \frac{\xi_{2}(s, r)}{\left(r^{2}-r_{1}^{2}\right)^{1 / 2}} d r d s \leq \frac{M}{(|a-t|+1)^{1 / 2+\gamma+\lambda}} .
$$

Combining (5.61) and (5.62), we obtain (5.58). To estimate the second term in the right hand side of (5.57), we note that

$$
\begin{aligned}
\frac{1}{\left\{\left(r-r_{1}\right)\left(r_{2}-r\right)\right\}^{1 / 2}} \leq & \frac{1}{\{(a+t-s-r+1)(r+s+a-t+1)\}^{1 / 2}} \\
& +\frac{1}{\{(a+t-s-r+1)(r-s-a+t+1)\}^{1 / 2}}
\end{aligned}
$$

Using (5.29) for the first term of (5.63) and the method above for the second term of (5.63), we get

$$
\iint_{\text {white }} \frac{\xi(s, r)}{\left\{\left(r-r_{1}\right)\left(r_{2}-r\right)\right\}^{1 / 2}} d r d s \leq \frac{M}{(|a-t|+1)^{1 / 2+\gamma+\lambda}} .
$$

Therefore from (5.57), (5.58) and (5.64) we obtain (5.24) for $i=5$. Consequently, we have proved (5.24).

Next, we prove (5.25). $\xi(s, r)$ and $\eta(s, r)$ are used again, but we do not consider $\xi_{1}(s, r)$ and $\xi_{2}(s, r)$ separately. Since $t>a$ when $D^{\prime \prime}$ is not empty, $r_{1}=t-a-s$.

(vi) Estimate of $I_{1}^{\prime \prime}$ 
By Proposition 5.3.II.(i),

$$
I_{1}^{\prime \prime} \leq M \iint_{\text {black }} \frac{r^{1 / 2}}{w(s, r)\left\{\left(r+r_{1}\right)\left(r_{2}-r\right)\right\}^{1 / 2}} \log \left[2+\frac{a r}{\left(r_{1}-r\right)\left(r+r_{2}\right)}\right] d r d s .
$$

In the domain $D^{\prime \prime}$, we use the following facts:

$$
\begin{aligned}
& \frac{1}{\left\{\left(r+r_{1}\right)\left(r_{2}-r\right)\right\}^{1 / 2}} \leq \frac{M}{\{(t-a)(a+t)\}^{1 / 2}} \text { for } r+s \leq(t-a) / 2, \\
& \frac{1}{w(s, r)} \leq \frac{M}{(|a-t|+1)^{1 / 2+\gamma+\lambda}} \eta(s, r) \text { for } r+s \geq(t-a) / 2, \\
& r_{2}-r \geq 2 a+\tilde{\delta} \text { for } r \leq r_{1}-\tilde{\delta}, \\
& \frac{a r}{\left(r_{1}-r\right)\left(r+r_{2}\right)} \leq \frac{r}{r_{1}-r} \leq M\left\{(t-a) \chi\left(2 r-r_{1}\right)+1\right\} \text { for } r \leq r_{1}-\tilde{\delta} .
\end{aligned}
$$

It follows from (5.66)-(5.69) and (5.3) that

$$
\begin{aligned}
I_{1}^{\prime \prime} \leq & \frac{M}{\{(t-a)(a+t)\}^{1 / 2}} \iint_{\substack{r+s \leq(t-a) / 2 \\
\text { black }}} \frac{r^{1 / 2}}{w(s, r)} d r d s \\
& +\frac{M}{(2 a+\tilde{\delta})^{1 / 2}(|a-t|+1)^{1 / 2+\gamma+\lambda}} \iint_{\substack{(t-a) / 2 \leq r+s \leq t-a-\tilde{\delta} \\
\text { black }}} \eta(s, r) \frac{r^{1 / 2}}{\left(r+r_{1}\right)^{1 / 2}} d r d s \\
& +\frac{M}{(|a-t|+1)^{1 / 2+\gamma+\lambda}} \cdot \\
& \cdot \iint_{\substack{\text { black } \\
t-a-\tilde{\delta} \leq r+s}} \eta(s, r) \frac{r^{1 / 2}}{\left\{\left(r+r_{1}\right)\left(r_{2}-r\right)\right\}^{1 / 2}} \log \left[2+\frac{a r}{\left(r_{1}-r\right)\left(r+r_{2}\right)}\right] d r d s .
\end{aligned}
$$

Moreover,

$$
\iint_{\substack{r+s \leq(t-a) / 2 \\ \text { black }}} \frac{r^{1 / 2}}{w(s, r)} d r d s \leq M \int_{0}^{t-a} \xi(s, 0) d s \int_{0}^{\tilde{\delta}} r^{1 / 2} d r \leq M \tilde{\delta}^{3 / 2}
$$

$$
\begin{aligned}
& \iint_{\substack{(t-a) / 2 \leq r+s \leq t-a-1 / 2 \\
\text { black }}} \eta(s, r) \frac{r^{1 / 2}}{\left(r+r_{1}\right)^{1 / 2}} d r d s \\
& \quad \leq M \int_{0}^{t-a} \frac{\eta(s, 0)}{\left(1+r_{1}\right)^{1 / 2}} d s \int_{0}^{1 / 2} d r \\
& \quad \leq M \int_{0}^{t-a} \frac{1}{(1+t-a-s)^{1 / 2}}\left\{\sum_{i=1}^{L} \frac{1}{(s+1)^{\tilde{\lambda}}\left(c_{i} s+1\right)}+\frac{1}{(s+1)^{\tilde{\lambda}+2 \gamma}}+\frac{1}{(s+1)^{1+\tilde{\lambda}}}\right\} d s
\end{aligned}
$$

$(5.72) \leq M$

because $\tilde{\lambda}+2 \gamma>1 / 2$ by (5.28). 
It remains to estimate the third term of the right-hand side of (5.70). We show that

$$
\begin{aligned}
& \int_{\left(r_{1}-\tilde{\delta}_{+}+\right.}^{r_{1}} \frac{r^{1 / 2}}{\left\{\left(r+r_{1}\right)\left(r_{2}-r\right)\right\}^{1 / 2}} \log \left[2+\frac{a r}{\left(r_{1}-r\right)\left(r+r_{2}\right)}\right] d r \\
& \quad \leq \frac{M}{(a+1)^{1 / 2}} \log [2+t-a]
\end{aligned}
$$

from which we obtain

$$
\begin{aligned}
& \iint_{\substack{t-a-\tilde{\delta} \leq r+s \\
\text { black }}} \eta(s, r) \frac{r^{1 / 2}}{\left\{\left(r+r_{1}\right)\left(r_{2}-r\right)\right\}^{1 / 2}} \log \left[2+\frac{a r}{\left(r_{1}-r\right)\left(r+r_{2}\right)}\right] d r d s \\
& \quad \leq M \int_{0}^{t-a} \eta\left(s, r_{1}\right) d s \int_{\left(r_{1}-\tilde{\delta}\right)_{+}}^{r_{1}} \frac{r^{1 / 2}}{\left\{\left(r+r_{1}\right)\left(r_{2}-r\right)\right\}^{1 / 2}} \log \left[2+\frac{a r}{\left(r_{1}-r\right)\left(r+r_{2}\right)}\right] d r \\
& \quad \leq \frac{M}{(a+1)^{1 / 2}} \log [2+t-a] .
\end{aligned}
$$

To prove (5.73), we consider the following two cases separately: (a) $1 \leq a$ and (b) $0<a<1$.

(a) $1 \leq a$

Since $r_{2}-r \geq r_{2}-r_{1} \geq a+1$, we have

$$
\begin{aligned}
& \int_{\left(r_{1}-\tilde{\delta}\right)_{+}}^{r_{1}} \frac{r^{1 / 2}}{\left\{\left(r+r_{1}\right)\left(r_{2}-r\right)\right\}^{1 / 2}} \log \left[2+\frac{a r}{\left(r_{1}-r\right)\left(r+r_{2}\right)}\right] d r \\
& \quad \leq \frac{1}{(a+1)^{1 / 2}} \int_{r_{1}-\tilde{\delta}}^{r_{1}} \log \left[2+\frac{r}{r_{1}-r}\right] d r .
\end{aligned}
$$

Hence by the way from which we derive (5.34), we have (5.73) for $a \geq 1$.

(b) $0<a<1$

Since $\log \left[2+a r /\left(r_{1}-r\right)\left(r+r_{2}\right)\right] \leq 1+a^{1 / 2} /\left(r_{1}-r\right)^{1 / 2}$ and $r_{2}-r \geq 2 a$, we have

$$
\begin{aligned}
& \int_{\left(r_{1}-\tilde{\delta}\right)_{+}}^{r_{1}} \frac{r^{1 / 2}}{\left\{\left(r+r_{1}\right)\left(r_{2}-r\right)\right\}^{1 / 2}} \log \left[2+\frac{a r}{\left(r_{1}-r\right)\left(r+r_{2}\right)}\right] d r \\
& \quad \leq \int_{r_{1}-\tilde{\delta}}^{r_{1}}\left\{\frac{1}{\left(r_{2}-r\right)^{1 / 2}}+\frac{1}{\left(r_{1}-r\right)^{1 / 2}}\right\} d r \\
& \quad \leq 4 \tilde{\delta}^{1 / 2} \leq 2 \sqrt{2} .
\end{aligned}
$$

Thus we obtain (5.73) for $0<a<1$.

Therefore it follows from (5.70)-(5.72) and (5.74) that

$$
I_{1}^{\prime \prime} \leq \frac{M \tilde{\delta}}{\{(t-a)(a+t)\}^{1 / 2}}+\frac{M}{(2 a+1 / 2)^{1 / 2}(|a-t|+1)^{1 / 2+\gamma+\lambda}}
$$




$$
\begin{aligned}
& +\frac{M}{(a+1)^{1 / 2}(|a-t|+1)^{1 / 2+\gamma+\lambda}} \log [2+t-a] \\
\leq & \frac{M}{(t-a+1)^{1 / 2}(t+a+1)^{1 / 2}}+\frac{M}{(a+1)^{1 / 2}(|a-t|+1)^{1 / 2+\gamma}} \\
\leq & \frac{M}{(a+1)^{1 / 2-\gamma}(a+t+1)^{\gamma}(|a-t|+1)^{1 / 2}} .
\end{aligned}
$$

(vii) Estimate of $I_{2}^{\prime \prime}$

By Proposition 5.3.II.(i),

$$
I_{2}^{\prime \prime} \leq M \iint_{\partial(\text { red })} \frac{r^{1 / 2}}{w(s, r)\left\{\left(r+r_{1}\right)\left(r_{2}-r\right)\right\}^{1 / 2}} \log \left[2+\frac{a r}{\left(r_{1}-r\right)\left(r+r_{2}\right)}\right] d \sigma .
$$

Moreover, by (5.66)-(5.69), we have

$$
\begin{aligned}
I_{2}^{\prime \prime} \leq & \frac{M}{\{(a+t+1)(t-a+1)\}^{1 / 2}} \iint_{\substack{s+r \leq(\text { (red) }) / 2 \\
s(s, r)}} \frac{r^{1 / 2}}{w(s)} d \sigma \\
& \quad+\frac{M}{(a+1)^{1 / 2}(|a-t|+1)^{1 / 2+\gamma+\lambda}} \log [2+t-a] \iint_{\substack{s+r \geq(\text { (red }) / 2 \\
\text { of }) / 2}} \eta(s, r) \frac{r^{1 / 2}}{\left(r+r_{1}\right)^{1 / 2}} d \sigma .
\end{aligned}
$$

Here we notice that $(t-a) / 2 \geq \tilde{\delta}=1 / 2$ when the domain red is not empty. We further see that

$$
\begin{aligned}
& \iint_{\substack{s+r \leq(\text { red }) \\
s-a) / 2}} \frac{r^{1 / 2}}{w(s, r)} d \sigma \leq M, \\
& \iint_{\substack{\partial(\text { red }) \\
s+r \geq(t-a) / 2}} \eta(s, r) \frac{r^{1 / 2}}{\left(r+r_{1}\right)^{1 / 2}} d \sigma \leq M .
\end{aligned}
$$

Hence from (5.76), (5.77) and (5.78) we obtain (5.25) for $i=2$.

(viii) Estimate of $I_{3}^{\prime \prime}$

By Proposition 5.3.II.(i),

$$
I_{3}^{\prime \prime} \leq M \iint_{\text {red }} \frac{1}{r^{1 / 2} w(s, r)\left\{\left(r+r_{1}\right)\left(r_{2}-r\right)\right\}^{1 / 2}} \log \left[2+\frac{a r}{\left(r_{1}-r\right)\left(r+r_{2}\right)}\right] d r d s .
$$

Further, by (5.66)-(5.69), we have

$$
\begin{aligned}
I_{3}^{\prime \prime} \leq & \frac{M}{\{(t-a+1)(a+t+1)\}^{1 / 2}} \iint_{\substack{\text { s+r } \leq(t-a) / 2 \\
\text { red }}} \frac{1}{r^{1 / 2} w(s, r)} d r d s \\
& \quad+\frac{M}{(a+1)^{1 / 2}(|a-t|+1)^{1 / 2+\gamma+\lambda}} \log [2+t-a] \iint_{\substack{s+r \geq(t-a) / 2 \\
\text { red }}} \frac{\eta(s, r)}{r^{1 / 2}\left(r+r_{1}\right)^{1 / 2}} d r d s .
\end{aligned}
$$


Since $r \geq \tilde{\delta}=1 / 2$ in the domain red, we have

$$
\begin{aligned}
\frac{1}{r^{1 / 2} w(s, r)} \leq & \frac{M \xi(s, r)}{(r+1)^{1 / 2}} \\
\leq & M\left\{\sum_{i=1}^{L} \frac{1}{(r+1)^{5 / 4}\left(\left|r-c_{i} s\right|+1\right)^{5 / 4}}+\frac{1}{(r+s+1)^{1+\gamma}(r+1)^{1+1 / 2-\gamma}}\right. \\
& \left.+\frac{1}{(r+1)^{5 / 4}(|r-s|+1)^{5 / 4}}\right\}
\end{aligned}
$$

for $(s, r) \in$ red. Therefore it follows that

$$
\iint_{\substack{s+r \leq(t-a) / 2 \\ r e d}} \frac{1}{r^{1 / 2} w(s, r)} d r d s \leq M
$$

Moreover,

$$
\begin{aligned}
\frac{\eta(s, r)}{r^{1 / 2}\left(r+r_{1}\right)^{1 / 2}} \leq & \frac{\eta(s, r)}{(r+1)^{1 / 2}\left(r+r_{1}+1\right)^{1 / 2}} \\
\leq & M\left\{\sum_{i=1}^{L} \frac{1}{(r+1)^{1+\tilde{\lambda} / 2}\left(\left|r-c_{i} s\right|+1\right)^{1+\tilde{\lambda} / 2}}\right. \\
& +\frac{1}{(r-s+t-a+1)^{1 / 2}(|r-s|+1)^{1 / 2+\min \{\gamma-\lambda, \tilde{\lambda} / 2\}}(r+1)^{1+\tilde{\lambda} / 2}} \\
& \left.+\frac{1}{(r+1)^{1+\tilde{\lambda} / 2}(|r-s|+1)^{1+\tilde{\lambda} / 2}}\right\}
\end{aligned}
$$

for $(s, r) \in$ red. Therefore it follows that

$$
\iint_{\substack{r+r \geq(t-a) / 2 \\ r^{1 / 2}\left(r+r_{1}\right)^{1 / 2}}} \frac{\eta(s, r)}{r^{1 / 2}} \leq M .
$$

Hence from (5.79), (5.80) and (5.81) we obtain (5.25) for $i=3$.

(ix) Estimate of $I_{4}^{\prime \prime}$

By Proposition 5.3.II.(ii),

$$
I_{4}^{\prime \prime} \leq M \iint_{\text {red }} \frac{r^{1 / 2}}{w(s, r)\left(r_{1}-r\right)\left\{\left(r+r_{1}\right)\left(r_{2}-r\right)\right\}^{1 / 2}} d r d s .
$$

Further, by (5.66), (5.67) and (5.68) we have

$$
\begin{aligned}
I_{4}^{\prime \prime} \leq & \frac{M}{\{(t-a+1)(a+t+1)\}^{1 / 2}} \iint_{\substack{s+r \leq(t-a) / 2 \\
r e d}} \frac{r^{1 / 2}}{w(s, r)\left(r_{1}-r\right)} d r d s \\
& \quad+\frac{M}{(a+1)^{1 / 2}(|a-t|+1)^{1 / 2+\gamma+\lambda}} \iint_{\substack{s+r \geq(t-a) / 2 \\
\text { red }}} \eta(s, r) \frac{r^{1 / 2}}{\left(r_{1}-r\right)\left(r+r_{1}\right)^{1 / 2}} d r d s .
\end{aligned}
$$


Both $r^{1 / 2} / w(s, r)\left(r_{1}-r\right)$ and $\eta(s, r) r^{1 / 2} /\left(r_{1}-r\right)\left(r+r_{1}\right)^{1 / 2}$ are bounded by $\eta(s, r) /\left(r_{1}-r\right)$. And since $r_{1}-r \geq \tilde{\delta}=1 / 2$ in the domain $r e d$, we have

$$
\begin{aligned}
\iint_{\text {red }} & \frac{\eta(s, r)}{r_{1}-r} d r d s \\
\leq & M \iint_{\text {red }} \frac{1}{r_{1}-r+1}\left\{\sum_{i=1}^{L} \frac{1}{(r+s+1)^{\tilde{\lambda} / 2}\left(\left|r-c_{i} s\right|+1\right)^{1+\tilde{\lambda} / 2}}\right. \\
& +\frac{1}{(r+s+1)^{\tilde{\lambda} / 2}(r+1)^{1+\tilde{\lambda} / 2}} \\
& \left.+\frac{1}{(r+s+1)^{\tilde{\lambda} / 2}(|r-s|+1)^{1+\tilde{\lambda} / 2}}\right\} d r d s \\
\leq & M .
\end{aligned}
$$

Therefore from (5.82) and (5.83) we have (5.25) for $i=4$. Consequently we have proved the estimate.

\section{Energy Estimates.}

In this section we prove

Proposition 6.1 Let $u=\left(u^{1}, \ldots, u^{m}\right) \in C^{\infty}\left([0, T) \times \mathbf{R}^{2} ; \mathbf{R}^{m}\right)$ be a solution of the following system of wave equations with $u(0, \cdot) \in C_{0}^{\infty}\left(\mathbf{R}^{2} ; \mathbf{R}^{m}\right)$.

$$
\partial_{t}^{2} u^{i}-c_{i}^{2} \Delta u^{i}=\sum_{j=1}^{m} \sum_{\alpha, \beta=0}^{2} C_{i j}^{\alpha \beta}(\partial u) \partial_{\alpha} \partial_{\beta} u^{j}+E_{i}(\partial u)
$$

Here, $C_{i j}^{\alpha \beta}, E_{i}(i, j=1,2, \ldots, m ; \alpha, \beta=0,1,2)$ are $C^{\infty}$-functions in $\{|\partial u|<1\}$, which satisfy the conditions (4.3)-(4.8).

Moreover we assume that

$$
\left|C_{i j}^{\alpha \beta}(\partial u)\right| \leq \frac{1}{4 m} \min \left\{1, c_{i}^{2}, c_{j}^{2}\right\} \quad \text { for } \quad|\partial u|<\delta_{1}
$$

and that there exists a positive number $T_{1}$ such that

$$
[\partial u]_{0}\left(T_{1}\right)<1 \text { and }|\partial u|_{0}\left(T_{1}\right)<\delta_{1}
$$


Then, we have the following energy estimates for $0<t<T_{1}$ :

$$
\begin{aligned}
\|\partial u(t)\|_{N}^{2} \leq & M_{N}\left\{\|\partial u(0)\|_{N}^{2}\right. \\
& \left.+\int_{0}^{t}(s+1)^{-1-\min \{1 / 3,2 \gamma\}}[\partial u(s)]_{0}^{2}\|\partial u(s)\|_{(N+1)(N+5)}^{2} d s\right\}
\end{aligned}
$$

(6.5) $\|\partial u(t)\|_{N}^{2} \leq M_{N} .\|\partial u(0)\|_{N}^{2}(t+1)^{M_{N}\left([\partial u]_{1}(t)\right)^{2}}$.

Proof. Since $\Omega$ commutes $\partial_{t}^{2}-c_{i}^{2} \Delta$,

$$
\partial_{t}^{2} \mathcal{D}^{A} u^{i}-c_{i}^{2} \Delta \mathcal{D}^{A} u^{i}=\sum_{j=1}^{m} \sum_{\alpha, \beta=0}^{2} \mathcal{D}^{A}\left\{C_{i j}^{\alpha \beta}(\partial u) \partial_{\alpha} \partial_{\beta} u^{j}\right\}+\mathcal{D}^{A} E_{i}(\partial u)
$$

We set

$$
\begin{aligned}
& a_{i j}^{\alpha \beta}= \begin{cases}1-C_{i i}^{00} & (\alpha=\beta=0, j=i) \\
-c_{i} c_{j} \delta_{\alpha \beta} \delta_{i j}-C_{i j}^{\alpha \beta} & \text { (otherwise) }\end{cases} \\
& w_{A}^{i}=\sum_{j=1}^{m} \sum_{\alpha, \beta=0}^{2}\left[\mathcal{D}^{A}\left\{C_{i j}^{\alpha \beta}(\partial u) \partial_{\alpha} \partial_{\beta} u^{j}\right\}-C_{i j}^{\alpha \beta}(\partial u) \partial_{\alpha} \partial_{\beta} \mathcal{D}^{A} u^{j}\right]+\mathcal{D}^{A} E_{i}(\partial u)
\end{aligned}
$$

Then from (6.6), (6.7) and (6.8) we have

$$
\sum_{j=1}^{m} \sum_{\alpha, \beta=0}^{2} a_{i j}^{\alpha \beta}(\partial u) \partial_{\alpha} \partial_{\beta} \mathcal{D}^{A} u^{j}=w_{A}^{i}
$$

Multiplying both side of (6.9) by $\partial_{t} \mathcal{D}^{A} u^{i}$ and using (4.3), we get

$$
\begin{aligned}
\sum_{i, j=1}^{m} & \sum_{\alpha, \beta=0}^{2}\left[\partial_{\alpha}\left\{2 a_{i j}^{\alpha \beta}(\partial u) \partial_{t} \mathcal{D}^{A} u^{i} \cdot \partial_{\beta} \mathcal{D}^{A} u^{j}\right\}-\partial_{t}\left\{a_{i j}^{\alpha \beta}(\partial u) \partial_{\alpha} \mathcal{D}^{A} u^{i} \cdot \partial_{\beta} \mathcal{D}^{A} u^{j}\right\}\right] \\
= & \sum_{i=1}^{m} 2 \partial_{t} \mathcal{D}^{A} u^{i} \cdot w_{A}^{i} \\
& \quad+\sum_{i, j=1}^{m} \sum_{\alpha \beta=0}^{2}\left\{\partial_{t} C_{i j}^{\alpha \beta}(\partial u) \partial_{\alpha} \mathcal{D}^{A} u^{i} \cdot \partial_{\beta} \mathcal{D}^{A} u^{j}-2 \partial_{\alpha} C_{i j}^{\alpha \beta}(\partial u) \partial_{t} \mathcal{D}^{A} u^{i} \cdot \partial_{\beta} \mathcal{D}^{A} u^{j}\right\}
\end{aligned}
$$

Integrating (6.10) over $[0, t] \times \mathbf{R}^{2}$, we have

$$
\begin{gathered}
\left\|\partial \mathcal{D}^{A} u(t)\right\|_{E}^{2}-\left\|\partial \mathcal{D}^{A} u(0)\right\|_{E}^{2} \\
=\int_{0}^{t} d s \iint_{\mathbf{R}^{2}}\left[\sum_{i=1}^{m} 2 \partial_{t} \mathcal{D}^{A} u^{i} \cdot w_{A}^{i}+\sum_{i, j=1}^{m} \sum_{\alpha, \beta=0}^{2}\left\{\partial_{t} C_{i j}^{\alpha \beta}(\partial u) \partial_{\alpha} \mathcal{D}^{A} u^{i} \cdot \partial_{\beta} \mathcal{D}^{A} u^{j}\right.\right. \\
\left.\left.-2 \partial_{\alpha} C_{i j}^{\alpha \beta}(\partial u) \partial_{t} \mathcal{D}^{A} u^{i} \cdot \partial_{\beta} \mathcal{D}^{A} u^{j}\right\}\right] d x
\end{gathered}
$$


where

$$
\begin{aligned}
\left\|\partial \mathcal{D}^{A} u(t)\right\|_{E}^{2}= & \iint_{\mathbf{R}^{2}} \sum_{i, j=1}^{m}\left\{a_{i j}^{00}(\partial u) \partial_{t} \mathcal{D}^{A} u^{i} \cdot \partial_{t} \mathcal{D}^{A} u^{j}(t, x)\right. \\
& \left.-\sum_{k, l=1}^{2} a_{i j}^{k l}(\partial u) \partial_{k} \mathcal{D}^{A} u^{i} \cdot \partial_{l} \mathcal{D}^{A} u^{j}(t, x)\right\} d x
\end{aligned}
$$

Notice that by (6.2) and (6.7) we have

$$
\frac{1}{M}\left\|\partial \mathcal{D}^{A} u(t)\right\|_{0} \leq\left\|\partial \mathcal{D}^{A} u(t)\right\|_{E} \leq M\left\|\partial \mathcal{D}^{A} u(t)\right\|_{0} .
$$

Therefore it follows that

$$
\left\|\partial \mathcal{D}^{A} u(t)\right\|_{0}^{2} \leq M\left(\left\|\partial \mathcal{D}^{A} u(0)\right\|_{0}^{2}+J_{A}^{(1)}+J_{A}^{(2)}\right)
$$

where

$$
\begin{aligned}
J_{A}^{(1)} & =\sum_{i=1}^{m} \int_{0}^{t} d s \iint_{\mathbf{R}^{2}}\left|\partial_{t} \mathcal{D}^{A} u^{i} \cdot w_{A}^{i}\right| d x \\
J_{A}^{(2)} & =\sum_{i, j=1}^{2} \sum_{\alpha, \beta=0}^{2} \int_{0}^{t} d s \iint_{\mathbf{R}^{2}}\left|\partial C_{i j}^{\alpha \beta}(\partial u)\right|\left|\partial \mathcal{D}^{A} u^{i}\right|\left|\partial \mathcal{D}^{A} u^{j}\right| d x
\end{aligned}
$$

We first prove the estimate (6.4). Since $|\partial u(s)|_{0}<1$, then by the assumption (4.4)-(4.8), we get

$$
\begin{aligned}
\left|\mathcal{D}^{B} C_{i j}^{\alpha \beta}(\partial u)\right| & \leq M_{B} \sum_{l=2}^{|B|+3} \sum_{j_{1}, \ldots, j_{l}=1}^{m} \sum_{\left|B_{1}\right|, \ldots,\left|B_{l}\right| \leq|B|} \delta_{i ; j j_{1} \cdots j_{l}} \prod_{k=1}^{l}\left|\mathcal{D}^{B_{k}} \partial u^{j_{k}}\right| \\
\left|\mathcal{D}^{A} E_{i}(\partial u)\right| & \leq M_{A} \sum_{l=3}^{|A|+4} \sum_{j_{1}, \ldots, j_{l}=1}^{m} \sum_{\left|A_{1}\right|, \ldots,\left|A_{l}\right| \leq|A|} \delta_{i ; j_{1} \cdots j_{l}} \prod_{k=1}^{l}\left|\mathcal{D}^{A_{k}} \partial u^{j_{k}}\right|
\end{aligned}
$$

Here we set

$$
\delta_{i ; j_{1} \cdots j_{l}}= \begin{cases}1-\delta_{i j_{1}} \delta_{i j_{2}} \delta_{i j_{3}} & (l=3) \\ 1 & (l \geq 4)\end{cases}
$$

Since $\left|\operatorname{det}\left(a_{i j}^{00}(\partial u)\right)_{i, j=1}^{m}\right| \geq 1 / 2^{m}$ from (6.2), we can solve the following simultaneous linear equation with respect to $\partial_{t}^{2} u^{i}$ :

$$
\sum_{j=1}^{m} a_{i j}^{00}(\partial u) \partial_{t}^{2} u^{j}=E_{i}(\partial u)-\sum_{j=1}^{m} \sum_{(\alpha, \beta) \neq(0,0)} a_{i j}^{\alpha \beta}(\partial u) \partial_{\alpha} \partial_{\beta} u^{j} \quad(i=1,2, \ldots, m) .
$$


Moreover, by (4.4), (4.5), (6.2) and Cramer's formula, we see

$$
\partial_{t}^{2} u^{l}=-\left\{\operatorname{det}\left(a_{i j}^{00}(\partial u)\right)_{i, j=1}^{m}\right\}^{-1} c_{l}^{2} \Delta u^{l}+\text { higher order terms }
$$

Therefore it follows from $(6.8),(6.14),(6.15)$ and (6.16) that

$(6.17) J_{A}^{(1)} \leq M_{A} \sum_{i=1}^{m} \sum_{l=3}^{|A|+5} \sum_{j_{1}, \ldots, j_{l}=1}^{m} \sum_{\left|A_{1}\right|, \ldots,\left|A_{l}\right| \leq|A|} \delta_{i ; j_{1} \ldots j_{l}} \int_{0}^{t} d s \iint_{\mathbf{R}^{2}} \prod_{k=1}^{l}\left|\mathcal{D}^{A_{k}} \partial u^{j_{k}}\right|\left|\mathcal{D}^{A} \partial u^{i}\right| d x$

Next, we consider $J_{A}^{(2)}$. By (4.4) and (4.7),

$$
\left|\partial C_{i j}^{\alpha \beta}(\partial u)\right| \leq M \sum_{k, l=1}^{m} \delta_{i ; j k l}\left|\partial u^{k}\right|\left|\partial^{2} u^{l}\right| .
$$

Therefore it follows from (6.16) that

$$
\begin{aligned}
J_{A}^{(2)} \leq & M\left\{\sum_{i, j, k, l=1}^{m} \sum_{h=1}^{2} \int_{0}^{t} d s \iint_{\mathbf{R}^{2}} \delta_{i ; j k l}\left|\partial u^{k}\right|\left|\partial_{h} \partial u^{l}\right|\left|\partial \mathcal{D}^{A} u^{i}\right|\left|\partial \mathcal{D}^{A} u^{j}\right| d x\right. \\
& \left.+\int_{0}^{t} d s \iint_{\mathbf{R}^{2}}|\partial u|^{3}\left|\partial \mathcal{D}^{A} u\right|^{2} d x\right\}
\end{aligned}
$$

Hence we find from (6.17) and (6.18) that

(6.19) $J_{A}^{(1)}+J_{A}^{(2)} \leq M_{A} \sum_{l=3}^{|A|+5} \sum_{j_{0}, \ldots, j_{l}=1}^{m} \sum_{\left|A_{0}\right|, \ldots,\left|A_{l}\right| \leq|A|+1} \delta_{j_{0} ; j_{1} \cdots j_{l}} \int_{0}^{t} d s \iint_{\mathbf{R}^{2}} \prod_{k=0}^{l}\left|\mathcal{D}^{A_{k}} \partial u^{j_{k}}\right| d x$.

By Hölder's inequality,

$$
\begin{aligned}
& \iint_{\mathbf{R}^{2}} \prod_{k=0}^{l}\left|\mathcal{D}^{A_{k}} \partial u^{j_{k}}(s, x)\right| d x \\
& \leq \quad\left\|\prod_{k=0}^{l-1} \mathcal{D}^{A_{k}} \partial u^{j_{k}}(s, \cdot)\right\|_{L^{2}\left(\mathbf{R}^{2}\right)}\left\|\mathcal{D}^{A_{l}} \partial u^{j_{l}}(s, \cdot)\right\|_{L^{2}\left(\mathbf{R}^{2}\right)} \\
& \leq \quad\left\|\prod_{k=0}^{l-1} w_{j_{k}}^{-(l-1) / l}(s,|\cdot|)\right\|_{L^{\infty}\left(\mathbf{R}^{2}\right)} \cdot \\
& \quad \cdot\left\|\prod_{k=0}^{l-1} w_{j_{k}}^{(l-1) / l}(s,|\cdot|) \mathcal{D}^{A_{k}} \partial u^{j_{k}}(s, \cdot)\right\|_{L^{2}\left(\mathbf{R}^{2}\right)}\left\|\mathcal{D}^{A_{l}} \partial u^{j_{l}}(s, \cdot)\right\|_{L^{2}\left(\mathbf{R}^{2}\right)} \\
& \leq\left\|\prod_{k=0}^{l-1} w_{j_{k}}^{-(l-1) / l}(s,|\cdot|)\right\|_{L^{\infty}\left(\mathbf{R}^{2}\right)} \cdot \\
& \quad \cdot \prod_{k=0}^{l-1}\left\|w_{j_{k}}^{(l-1) / l}(s,|\cdot|) \mathcal{D}^{A_{k}} \partial u^{j_{k}}(s, \cdot)\right\|_{L^{2 l}\left(\mathbf{R}^{2}\right)} \| \mathcal{D}^{A_{l} \partial u^{j_{l}}(s, \cdot) \|_{L^{2}\left(\mathbf{R}^{2}\right)}} .
\end{aligned}
$$

35 
Without loss of generality we may suppose that $j_{0}=j_{1}=j_{2}$ does not hold for $l=3$. Therefore, it follows from (3.1) that

$$
\left\|\prod_{k=0}^{l-1} w_{j_{k}}^{-(l-1) / l}(s,|\cdot|)\right\|_{L^{\infty}\left(\mathbf{R}^{2}\right)} \leq M(s+1)^{-1-\min \{1 / 3,2 \gamma\}} .
$$

In order to estimate (6.20), we need Gagliardo-Nirenberg inequality:

Lemma 6.1 Let $f \in C_{0}^{\infty}\left(\mathbf{R}^{2}\right),|A|=i \leq k$. Then,

$$
\left\|\mathcal{D}^{A} f\right\|_{L^{r k / i}(X)} \leq M_{A}\|f\|_{L^{\infty}(X)}^{1-i / k}\left(\sum_{|B| \leq k}\left\|\mathcal{D}^{B} f\right\|_{L^{r}(X)}\right)^{i / k}
$$

where

$$
X=\mathbf{R}^{2} \text { or }\left\{x\left|x \in \mathbf{R}^{2}, n \leq\right| x \mid \leq n+1\right\} \quad(n=0,1,2, \ldots) .
$$

For the proof, see [6], Appendix.

Since

$$
\frac{1}{M} w_{j}(s, n) \leq w_{j}(s, r) \leq M w_{j}(s, n)
$$

for $n \leq r \leq n+1$, we find from Lemma 6.1 that

$$
\begin{aligned}
&\left\|w_{j_{k}}(s,|\cdot|)^{(l-1) / l} \mathcal{D}^{A_{k}} \partial u^{j_{k}}(s, \cdot)\right\|_{L^{2 l}\left(\mathbf{R}^{2}\right)}^{2 l} \\
& \leq M \sum_{n=0}^{\infty} w_{j_{k}}(s, n)^{2(l-1)}\left\|\mathcal{D}^{A_{k}} \partial u^{j_{k}}\right\|_{L^{2 l}(\{n \leq|x| \leq n+1\})}^{2 l} \\
& \leq M_{A} \sum_{n=0}^{\infty} w_{j_{k}}(s, n)^{2(l-1)}\left\|\partial u^{j_{k}}(s, \cdot)\right\|_{L^{\infty}(\{n \leq|x| \leq n+1\})}^{2(l-1)} \\
& \cdot\left(\sum_{|B| \leq l\left|A_{k}\right|}\left\|\mathcal{D}^{B} \partial u^{j_{k}}(s, \cdot)\right\|_{L^{2}(\{n \leq|x| \leq n+1\})}\right)^{2} \\
& \leq M_{A}[\partial u(s)]_{0}^{2(l-1)}\|\partial u(s)\|_{l\left|A_{k}\right|}^{2}
\end{aligned}
$$

Therefore, from (6.3), (6.19)-(6.23) and (6.13), we get (6.4).

Next, we prove (6.5). For the proof, we use the following two lemmas:

Lemma 6.2 Let $f, g \in C_{0}^{\infty}\left(\mathbf{R}^{2}\right)$. Then,

$$
\left\|\mathcal{D}^{A}(f g)-f \mathcal{D}^{A} g\right\|_{0} \leq M\left(|f|_{1}\|g\|_{|A|-1}+|g|_{0}\|f\|_{|A|}\right) .
$$


Lemma 6.3 Let $f=\left(f_{1}, \ldots, f_{r}\right) \in C_{0}^{\infty}\left(\mathbf{R}^{2} ; \mathbf{R}^{r}\right)$ and let $\omega=\omega(f)$ be a $C^{\infty}$-function that satisfies

$$
|\omega(f)| \leq M|f|^{q}
$$

Then

$$
\left\|\mathcal{D}^{A}(\omega \circ f)\right\|_{L^{p}\left(\mathbf{R}^{2}\right)} \leq M|f|_{L^{\infty}\left(\mathbf{R}^{n}\right)}^{q-1} \sum_{|B| \leq|A|}\left\|\mathcal{D}^{B} f\right\|_{L^{p}\left(\mathbf{R}^{2}\right)}
$$

See [6], Appendix for the proof of Lemma 6.2, and [10], Proposition A2 for Lemma 6.3.

By these lemmas,

$$
\left\|w_{A}^{i}\right\|_{L^{2}\left(\mathbf{R}^{2}\right)} \leq M_{A}|\partial u(s)|_{0}|\partial u(s)|_{1}\|\partial u(s)\|_{|A|} .
$$

Therefore from (6.18) and (6.25) we get

$$
J_{A}^{(1)}+J_{A}^{(2)} \leq M_{A} \int_{0}^{t}|\partial u(s)|_{0}|\partial u(s)|_{1}\|\partial u(s)\|_{|A|}^{2} d s .
$$

Further, it follows from (6.13) and (6.26) that

$$
\|\partial u(t)\|_{N}^{2} \leq M_{N}\left\{\|\partial u(0)\|_{N}^{2}+\int_{0}^{t}|\partial u(s)|_{0}|\partial u(s)|_{1}\|\partial u(s)\|_{N}^{2} d s\right\} .
$$

Hence by Gronwall's lemma we find

$$
\|\partial u(t)\|_{N}^{2} \leq M\|\partial u(0)\|_{N}^{2} \exp \left(M_{N} \int_{0}^{t}|\partial u(s)|_{0}|\partial u(s)|_{1} d s\right) .
$$

Since

$$
|\partial u(s)|_{0}|\partial u(s)|_{1} \leq M_{N}(s+1)^{-1}[\partial u(s)]_{1}^{2}
$$

we obtain (6.5) from (6.27).

\section{Proof of the Theorem.}

Making use of the method in [1] and [5], we find that a solution $u(t, x)$ to $(4.1)$ is unique and $u(t, \cdot)(t \geq 0)$ has compact support. The local existence theorem of a solution to (4.1) has proved in [4] and [7]. 
Let $u(t, x)$ be a $C^{\infty}$-solution to $(4.1)$ in $[0, T) \times \mathbf{R}^{2}$. We write $u$ as

$$
u=u_{0}+u_{1}
$$

where $u_{0}$ is the solution of the initial value problem

$$
\left\{\begin{array}{l}
\partial_{t}^{2} u_{0}^{i}-c_{i}^{2} \Delta u_{0}^{i}=0 \\
u_{0}^{i}(0, \cdot)=\varepsilon f_{i}, \partial_{t} u_{0}^{i}(0, \cdot)=\varepsilon g_{i} \quad(i=1,2, \ldots, m),
\end{array}\right.
$$

and $u_{1}$ is the solution of the initial value problem

$$
\left\{\begin{array}{l}
\partial_{t}^{2} u_{1}^{i}-c_{i}^{2} \Delta u_{1}^{i}=F_{i}\left(\partial u, \partial^{2} u\right) \\
u_{1}^{i}(0, \cdot)=\partial_{t} u_{1}^{i}(0, \cdot)=0 \quad(i=1,2, \ldots, m) .
\end{array}\right.
$$

In [2], R. Glassey has proved by the method in W. von Wahl [14] that

$$
\left|u_{0}^{i}(t, x)\right| \leq \frac{M \varepsilon}{\left\{\left(|x|+c_{i} t+1\right)\left(|| x\left|-c_{i} t\right|+1\right)\right\}^{1 / 2}} .
$$

Here $M$ depends on $L^{1}$-norm of $f_{i}, \partial f_{i}$ and $g_{i}$.

We set

$$
\begin{aligned}
\frac{1}{\tilde{w}_{i}(s, r)}= & \sum_{j \neq i} \frac{1}{(r+s+1)\left(\left|r-c_{j} s\right|+1\right)}+\frac{1}{(r+s+1)^{1+2 \gamma}(r+1)^{1-2 \gamma}} \\
& +\frac{1}{(r+s+1)^{4 / 3}\left(\left|r-c_{i} s\right|+1\right)^{2 / 3}} .
\end{aligned}
$$

Then $\tilde{w}_{i}$ satisfies (5.20) and (5.21). By Proposition 5.4, we get

$$
\begin{aligned}
{\left[\partial u_{1}(t)\right]_{N} \leq } & M_{N}\left\{\sum_{i=1}^{m} \sum_{|A| \leq N+3} \sup _{0<s<t}\left\|\tilde{w}_{i}(s,|\cdot|) \mathcal{D}^{A} F_{i}\left(\partial u, \partial^{2} u\right)(s, \cdot)\right\|_{L^{2}\left(\mathbf{R}^{2}\right)}\right. \\
& \left.+\sum_{i=1}^{m} \sum_{|A| \leq N+2} \sup _{0<s<t}\left\|\tilde{w}_{i}(s,|\cdot|) \partial_{t} \mathcal{D}^{A} F_{i}\left(\partial u, \partial^{2} u\right)(s, \cdot)\right\|_{L^{2}\left(\mathbf{R}^{2}\right)}\right\} .
\end{aligned}
$$

Since $[\partial u(t)]_{0}$ is continuous, we can take for $0<\varepsilon<\varepsilon_{1}$ a positive number $T_{1}$ such that the condition (6.3) holds, provided $\varepsilon_{1}$ is small enough. We suppose $0<\varepsilon<\varepsilon_{1}$ and set

$$
\tilde{T}_{1}=\sup \left\{T_{1} \mid(6.3) \text { holds. }\right\}
$$


Let $0<t<\tilde{T}_{1}$ in the following. In particular, $|\partial u|_{0}(t)<1$. Then by $(6.16)$, we have

$$
\begin{gathered}
\left|\mathcal{D}^{A} F_{i}\left(\partial u, \partial^{2} u\right)\right| \leq M_{A} \sum_{l=3}^{|A|+6} \sum_{j_{1}, \ldots, j_{l}=1}^{m} \sum_{\substack{\left|A_{h}\right| \leq|A|+1 \\
(h=1,2, \ldots, l)}} \delta_{i ; j_{1} \cdots j_{l}} \prod_{k=1}^{l}\left|\mathcal{D}^{A_{k}} \partial u^{j_{k}}\right| \\
\left|\partial_{t} \mathcal{D}^{A} F_{i}\left(\partial u, \partial^{2} u\right)\right| \leq M_{A} \sum_{l=3}^{|A|+8} \sum_{j_{1}, \ldots, j_{l}=1}^{m} \sum_{\substack{\left|A_{h}\right| \leq|A|+2 \\
(h=1,2, \ldots, l)}} \delta_{i_{i} ; j_{1} \cdots j_{l}} \prod_{k=1}^{l}\left|\mathcal{D}^{A_{k}} \partial u^{j_{k}}\right| .
\end{gathered}
$$

Hence from (7.5), (7.6) and (7.7) we get

(7.8) $\left[\partial u_{1}(t)\right]_{N}$

$$
\leq M_{N} \sum_{l=3}^{N+10} \sum_{j_{1}, \ldots, j_{l}=1}^{m} \sum_{\substack{A_{h} \mid \leq N+4 \\(h=1,2, \ldots, l)}} \delta_{i ; j_{1} \cdots j_{l}} \sup _{0<s<t}\left\|\tilde{w}_{i}(s,|\cdot|) \prod_{k=1}^{l} \mathcal{D}^{A_{k}} \partial u^{j_{k}}(s, \cdot)\right\|_{L^{2}\left(\mathbf{R}^{2}\right)} .
$$

We notice that

$$
\frac{1}{\left(w_{j_{1}} w_{j_{2}} w_{j_{3}}\right)^{2 / 3}(s, r)} \leq \frac{M}{\tilde{w}_{i}(s, r)}
$$

provided $j_{1}=j_{2}=j_{3}=i$ does not hold. Thus we get

$$
\delta_{i ; j_{1} \cdots j_{l}} \tilde{w}_{i} \leq M\left(w_{j_{1}} \cdots w_{j_{l}}\right)^{(l-1) / l}
$$

By (7.9) and Hölder's inequality,

$$
\begin{aligned}
\delta_{i ; j_{1} \cdots j_{l}}\left\|\tilde{w}_{i}(s,|\cdot|) \prod_{k=1}^{l} \mathcal{D}^{A_{k}} \partial u^{j_{k}}(s, \cdot)\right\|_{L^{2}\left(\mathbf{R}^{2}\right)} & \leq M\left\|\prod_{k=1}^{l} w_{j_{k}}^{(l-1) / l}(s,|\cdot|) \mathcal{D}^{A_{k}} \partial u^{j_{k}}(s, \cdot)\right\|_{L^{2}\left(\mathbf{R}^{2}\right)} \\
& \leq M \prod_{k=1}^{l}\left\|w_{j_{k}}^{(l-1) / l}(s,|\cdot|) \mathcal{D}^{A_{k}} \partial u^{j_{k}}(s, \cdot)\right\|_{L^{2 l}\left(\mathbf{R}^{2}\right)}
\end{aligned}
$$

Hence by $(7.8),(7.10)$ and $(6.23)$ we have

$$
\left[\partial u_{1}(t)\right]_{N} \leq M_{N} \sup _{0<s<t}[\partial u(s)]_{0}^{2}\|\partial u(s)\|_{N_{1}}
$$

where $N_{1}=(N+10)(N+4)$. Therefore it follows from (7.1), (7.4) and (7.11) that

$$
[\partial u(t)]_{N} \leq M_{N}\left\{\varepsilon+\sup _{0<s<t}[\partial u(s)]_{0}^{2}\|\partial u(s)\|_{N_{1}}\right\} .
$$


By Proposition 6.1,

(7.14) $\|\partial u(\tau)\|_{\left(N_{1}+1\right)\left(N_{1}+5\right)} \leq M_{N}(\tau+1)^{M_{N}[\partial u]_{1}^{2}(\tau)}$.

We fix the constant $M_{N}$ in (7.12), (7.13) and (7.14) so that

$$
M_{N} \geq \max \left\{8,2 / \min \{1 / 3,2 \gamma\},\left(2 / \delta_{1}\right)^{1 / 2}\right\}
$$

We take $\varepsilon_{0}$ to be

$$
0<\varepsilon_{0}<\min \left\{1 / 4 M_{N}^{3}, \varepsilon_{1}\right\}
$$

Moreover, we suppose that $\varepsilon_{0}$ is small enough to define the following $T_{0}$ for $0<\varepsilon<\varepsilon_{0}$ :

$$
T_{0}=\sup \left\{t \mid[\partial u]_{N}(t) \leq 4 \varepsilon M_{N}\right\} .
$$

Suppose that $0<t<T_{0}$. Then,

$$
\begin{aligned}
& {[\partial u(t)]_{0} \leq 4 \varepsilon M_{N} \leq 1 / M_{N}^{2} \leq 1 / 2} \\
& |\partial u(t)|_{0} \leq 1 / M_{N}^{2} \leq \delta_{1} / 2 .
\end{aligned}
$$

Therefore $T_{0} \leq \tilde{T}_{1}$. Then for $0<\varepsilon<\varepsilon_{0}$ and $0<t<T_{0}$,

$$
\begin{aligned}
& {[\partial u]_{1}^{2}(t) \leq\left(4 \varepsilon M_{N}\right)^{2} \leq 1 / M_{N}^{2}} \\
& -\min \{1 / 3,2 \gamma\}+M_{N}[\partial u]_{1}^{2}(\tau) \leq-\min \{1 / 3,2 \gamma\} / 2 .
\end{aligned}
$$

Here from (7.13), (7.14) and (7.15) we get

$$
\begin{aligned}
\|\partial u(s)\|_{N_{1}}^{2} & \leq M_{N}\left\{1+\frac{1}{M_{N}^{2}} \cdot M_{N} \int_{0}^{s}(\tau+1)^{-1-\min \{1 / 3,2 \gamma\} / 2} d \tau\right\} \\
& \leq 2 M_{N}
\end{aligned}
$$

Therefore it follows from (7.12) and (7.16) that

$$
\begin{aligned}
{[\partial u(t)]_{N} } & \leq M_{N}\left\{\varepsilon+\sup _{0<s<t}[\partial u(s)]_{0} \cdot 4 \varepsilon M_{N} \cdot\left(2 M_{N}\right)^{1 / 2}\right\} \\
& \leq M_{N} \varepsilon+\frac{1}{2} \sup _{0<s<t}[\partial u(s)]_{0} \\
& \leq M_{N} \varepsilon+\frac{1}{2} \sup _{0<s<T_{0}}[\partial u(s)]_{N}
\end{aligned}
$$


which implies

$$
\sup _{0<t<T_{0}}[\partial u(t)]_{N} \leq 2 M_{N} \varepsilon
$$

Therefore $T_{0}$ cannot be finite, and we complete the proof of the theorem.

\section{References}

[1] R. Agemi, Blow-up of solutions to nonlinear wave equation in two space dimensions, Manuscripta Math. 73 (1991), 153-162.

[2] R. T. Glassey, Existence in the large for $\square u=F(u)$ in two space dimensions, Math. Z. 178 (1981), 233-261.

[3] F. John, Formation of singularities in one-dimensional nonlinear wave propagation. Comm. Pure Appl. Math. 27 (1974), 377-405.

[4] F. John, Delayed singularity formation in solutions of nonlinear wave equations in higher dimensions. Comm. Pure Appl. Math. 29 (1976), 649-682.

[5] F. John, Nonlinear wave equations, Formation of singuralities, Pitcher lectures in the Math. Sci., Lehigh Univ., American Math. Soc., 1990

[6] F. John and S. Klainerman, Almost global existence to nonlinear wave equations in the three space dimensions, Comm. Pure Appl. Math. 37 (1984), 443-455.

[7] T. Kato, The Cauchy problem for quasilinear symmetric hyperbolic systems, Arch. Rational Mech. Anal.58 (1975), 181-205.

[8] S. Klainerman, Global existence for nonlinear wave equations, Comm. Pure Appl. Math. 33 (1980), 43-101.

[9] S. Klainerman, The null condition and global existence to nonlinear wave equations, Lectures in Appl. Math. 23 (1986), Amer. Math. Soc., 293-326. 
[10] M. Kovalyov, Long-time behaviour of solutions of a system on non-linear wave equations, Comm. in PDE., 12 (1987), 471-501.

[11] M. Kovalyov, Resonance-type behaviour in a system of nonlinear wave equations, J. Differential Equations 77 (1989), 73-83.

[12] Li Ta-tsien, Kong De-xing and Zhou Yi, Global classical solutions for quasilinear nonstrictly hyperbolic systems, preprint.

[13] T. Sideris, The null condition and global existence of nonlinear elastic waves, Invent. math. 123, (1996), 323-342.

[14] W. von Wahl, L $L^{P}$-decay rates for homogeneous wave equations, Math. Z. 120 (1971), 93-106. 